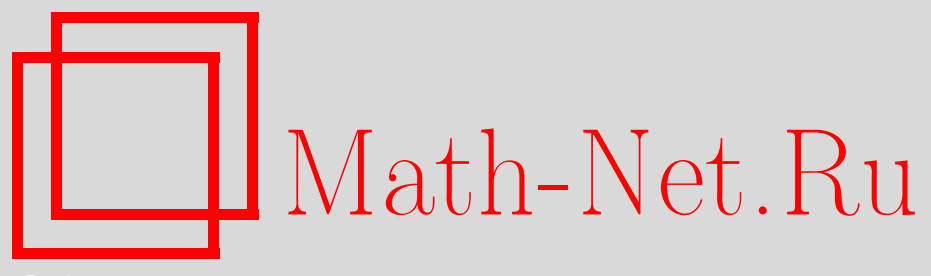

В. И. Черноусов, А. С. Рапинчук, И. А. Рапинчук, Алгебры с делением, имеющие одинаковые максимальные подполя, УМН, 2015, том 70, выпуск 1, 89-122

DOI: https://doi.org/10.4213/rm9649

Использование Общероссийского математического портала Math-Net.Ru подразумевает, что вы прочитали и согласны с пользовательским соглашением http://www . mathnet.ru/rus/agreement

Параметры загрузки:

IP : 52.23 .180 .231

26 апреля 2023 г., 06:05:16

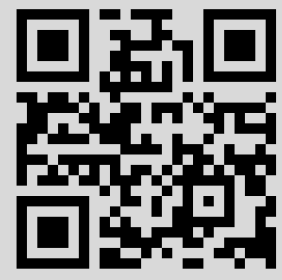




\section{Алгебры с делением, имеющие одинаковые максимальные подполя}

\section{В. И. Черноусов, А. С. Рапинчук, И. А. Рапинчук}

В статье приводится обзор недавних результатов по проблеме характеризации конечномерных алгебр с делением в терминах их максимальных подполей. Мы также обсуждаем обобщения этой проблемы и ее различные приложения. В последнем разделе рассматривается более широкая постановка задачи в контексте абсолютно простых алгебраических групп.

Библиография: 51 название.

Ключевые слова: алгебра с делением, неразветвленная группа Брауэра, полупростые алгебраические группы.

DOI: $10.4213 / \mathrm{rm} 9649$

\section{СОДЕРЖАНИЕ}

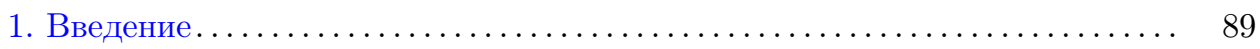

2. Мотивировки ........................................ 92

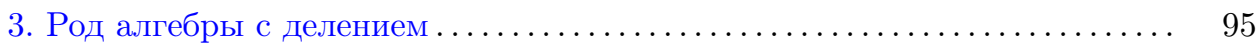

4. Ветвление алгебр с делением ............................. 100

5. Некоторые модификации понятия рода ..................... 109

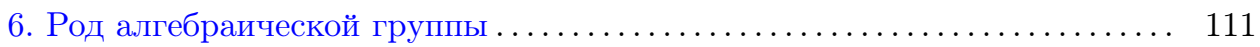

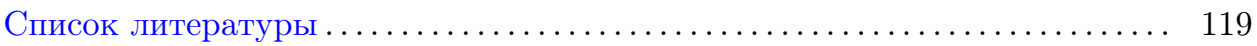

\section{1. Введение}

Цель этой статьи - дать обзор недавних работ по проблеме характеризации конечномерных алгебр с делением в терминах их максимальных подполей и, более общо, простых алгебраических групп в терминах их максимальных торов. Мы рассматриваем следующий вопрос, а также его вариации, которые будут обсуждены позже.

(†) Что можно сказать о двух конечномерных иентральных алгебрах с делением $D_{1}$ и $D_{2}$ над полем $K$ при условии, что $D_{1}$ и $D_{2}$ имеют одинаковые (с точностью до изоморфизма) максимальные подполя?

(С) В. И. Черноусов, А. С. РАПинчук, И. А. РАПинчук, 2015 
Точнее, условие, наложенное на $D_{1}$ и $D_{2}$, означает, что они имеют одинаковую степень $n$ над $K$ (т. е. $\operatorname{dim}_{K} D_{1}=\operatorname{dim}_{K} D_{2}=n^{2}$ ) и расширение $P / K$ степени $n$ может быть вложено в $D_{1}$ над $K$ тогда и только тогда, когда оно может быть вложено в $D_{2}$.

Напомним, что расширение $F$ поля $K$ называется полем разложения центральной простой алгебры $A$ степени $n$, если существует изоморфизм $F$-алгебр $A \otimes_{K} F \simeq M_{n}(F)$. Кроме того, если $A$ - алгебра с делением, то поля разложения степени $n$ над $K$ - это в точности максимальные подполя в $A$ (см., например, [15; теорема 4.4]). Поскольку поля разложения и/или максимальные подполя $K$-алгебры с делением $D$ или, более общо, любой конечномерной центральной простой алгебры являются основой для анализа ее структуры, естественно возникает вопрос, в какой степени эти поля на самом деле определяют $D$. В случае, когда рассматриваются все поля разложения, ответ на этот вопрос был получен в 1955 г. Ш. Амицуром [2]:

Если $A_{1}$ и $A_{2}$ - конечномерные централъные простые алгебры над полем $K$, имеющие одинаковые поля разложения (т.е. расширение поля $F / K$ расщепляет $A_{1}$ тогда и только тогда, когда оно расщепляет $\left.A_{2}\right)$, то классы $\left[A_{1}\right]$ u $\left[A_{2}\right]$ в группе Брауэра $\operatorname{Br}(K)$ порождают одну и ту же подгруппу: $\left\langle\left[A_{1}\right]\right\rangle=\left\langle\left[A_{2}\right]\right\rangle$.

В доказательстве этой теоремы (см. [2], [21; гл. 5]) используются так называемые поля разложения общего типа, которые имеют бесконечную степень над $K$. Однако ситуация резко меняется, если допускаются только конечномерные поля разложения. Это показывает следующий пример кубической алгебры с делением над $\mathbb{Q}$.

Сначала напомним теорему Альберта-Брауэра-Хассе-Нётер, которая утверждает, что для глобального поля $K$ имеется точная последовательность

$$
0 \rightarrow \operatorname{Br}(K) \rightarrow \bigoplus_{v \in V^{K}} \operatorname{Br}\left(K_{v}\right) \stackrel{\sum \operatorname{inv}_{v}}{\longrightarrow} \mathbb{Q} / \mathbb{Z} \rightarrow 0
$$

где $V^{K}$ - множество всех (классов эквивалентности) нормирований поля $K$, $K_{v}$ - пополнение $K$ относительно $v$ и $\operatorname{inv}_{v}$ - так называемый инвариант, задающий изоморфизм $\operatorname{Br}\left(K_{v}\right) \simeq \mathbb{Q} / \mathbb{Z}$, когда $v$ - неархимедово нормирование, и изоморфизм $\operatorname{Br}\left(K_{v}\right) \simeq(1 / 2) \mathbb{Z} / \mathbb{Z}$, когда $v$ вещественно (см., например, [1; гл. VII, § 9.6] и $[34 ; \S 18.4]$ для числовых полей, $[21 ; \S 6.5]$ для функциональных полей; историю вопроса см. в [42]). Зафиксируем целое $r \geqslant 2$ и возьмем $r$ различных простых чисел $p_{1}, \ldots, p_{r}$. Пусть $\varepsilon=\left(\varepsilon_{1}, \ldots, \varepsilon_{r}\right)$ - произвольный набор из $r$ элементов, где $\varepsilon_{i} \in\{ \pm 1\}$ и $\sum_{i=1}^{r} \varepsilon_{i} \equiv 0(\bmod 3)$. Согласно $(\mathrm{ABHN})$, существует кубическая алгебра с делением $D(\varepsilon)$ над $\mathbb{Q}$ со следующими локальными инвариантами (рассматриваемыми как элементы $\mathbb{Q} / \mathbb{Z}$ ):

$$
\operatorname{inv}_{p} D(\varepsilon)= \begin{cases}\frac{\varepsilon_{i}}{3}, & p=p_{i}, i=1, \ldots, r \\ 0, & \left.p \notin\left\{p_{1}, \ldots, p_{r}\right\} \text { (включая } p=\infty\right) .\end{cases}
$$


Тогда для любых двух наборов $\varepsilon^{\prime} \neq \varepsilon^{\prime \prime}$ такого вида алгебры $D\left(\varepsilon^{\prime}\right)$ и $D\left(\varepsilon^{\prime \prime}\right)$ имеют одинаковые конечномерные поля разложения и, следовательно, одни и те же максимальные подполя (см. [34; $\$ 18.4$, следствие b]), но не являются изоморфными. Ясно, что количество таких наборов $\varepsilon$ растет с ростом $r$, поэтому этот метод позволяет построить произвольно большое (но конечное) число попарно неизоморфных кубических алгебр с делением над $\mathbb{Q}$, имеющих одинаковые максимальные подполя (в то же время циклические подгруппы $\langle[D(\varepsilon)]\rangle$ имеют порядок 3).

Подобная конструкция применима для алгебр с делением любой степени $d>2$. С другой стороны, из $(\mathrm{ABHN})$ следует, что тело кватернионов $D$ над числовым полем определяется с точностью до изоморфизма своим набором максимальных подполей (см. раздел 3). Таким образом, рассмотрение только конечномерных полей разложения (и, в частности, максимальных подполей) делает вопрос $(\dagger)$ весьма нетривиальным и интересным.

Для анализа алгебр с делением, имеющих одинаковые максимальные подполя, удобно ввести следующее понятие. Пусть $D$ - конечномерная центральная алгебра с делением над полем $K$. Мы определяем род $D$ следующим образом:

$$
\begin{array}{r}
\operatorname{gen}(D)=\left\{\left[D^{\prime}\right] \in \operatorname{Br}(K) \mid D^{\prime}-\right.\text { алгебра с делением, имеющая те же } \\
\quad \text { максимальные подполя, что и } D\} .
\end{array}
$$

Среди различных вопросов, связанных с понятием рода, мы остановимся в данной работе на следующих двух.

Вопрос 1. Когда $\operatorname{gen}(D)$ сводится к одному элементу (m.е. когда D определяется своими максимальными подполями однозначно с точностью до изоморфизма)?

\section{Вопрос 2. Когда множество gen $(D)$ конечно?}

Структура статьи следующая. Мы представим имеющиеся результаты по вопросам 1 и 2 в разделе 3 ; общий подход к их изучению, основанный на анализе ветвлений в алгебрах с делением, будет изложен в разделе 4. Далее в разделе 5 мы кратко рассмотрим несколько других полезных понятий рода, в том числе локальный род и односторонний род. Наконец, в разделе 6 мы дадим обзор продолжающихся исследований, целью которых является распространение анализа алгебр с делением, имеющих одинаковые максимальные подполя, на алгебраические группы с одними и теми же классами изоморфных или изогенных максимальных торов.

Обозначения. Для поля $K$ с дискретным нормированием $v$ обозначим через $K_{v}$ его пополнение относительно $v$; пусть $\mathscr{O}_{v} \subset K_{v}-$ кольцо нормирования и $\bar{K}_{v}$ - соответствующее поле вычетов. Далее, если $K$ - числовое поле, то $V^{K}$ будет обозначать множество (классов эквивалентности) нормирований поля $K$, a $V_{\infty}^{K}$ - подмножество (классов) архимедовых нормирований. Наконец, для поля $K$ характеристики $\neq 2$ и произвольной пары ненулевых элементов $a, b \in K^{\times}$ 
обозначим через $D=\left(\frac{a, b}{K}\right)$ соответствующую алгебру кватернионов, т. е. четырехмерную $K$-алгебру с базисом $1, i, j, k$ и умножением, определенным следующим образом:

$$
i^{2}=a, \quad j^{2}=b, \quad i j=k=-j i .
$$

\section{2. Мотивировки}

В этом разделе мы опишем две мотивировки, которые естественным образом привели к рассмотрению вопросов в духе $(\dagger)$. Первая демонстрирует связь с теорией квадратичных форм, а вторая, наиболее важная для нас, вытекает из задач в дифференциальной геометрии.

Пусть $K$ - поле характеристики $\neq 2$. Алгебре кватернионов $D=\left(\frac{a, b}{K}\right)$ поставим в соответствие квадратичную форму

$$
q(x, y, z)=a x^{2}+b y^{2}-a b z^{2} .
$$

Заметим, что с точностью до знака эта форма совпадает с приведенной нормой на пространстве чистых кватернионов, из чего следует, что для любого $d \in$ $K^{\times} \backslash\left(K^{\times}\right)^{2}$ квадратичное расширение $K(\sqrt{d}) / K$ вкладывается в $D$ тогда и только тогда, когда $q$ представляет $d$ (см. [21; замечание 1.1.4]).

Теперь предположим, что даны две алгебры кватернионов с делением $D_{1}$ и $D_{2}$ с соответствующими квадратичными формами $q_{1}$ и $q_{2}$. Тогда эти алгебры имеют одинаковые максимальные подполя в том и только том случае, когда $q_{1}$ и $q_{2}$ представляют одинаковые элементы над $K$. С другой стороны, хорошо известно, что $D_{1}$ и $D_{2}$ являются $K$-изоморфными в точности тогда, когда $q_{1}$ и $q_{2}$ эквивалентны над $K$ (см. [34; $\S 1.7$, предложение]). Итак, $(\dagger)$ приводит нас к следующему естественному вопросу о квадратичных формах.

Предположим, что $q_{1}$ и $q_{2}$ - тернарные формы с определителем -1, которые представляют одни и те же элементы над $K$. Обязательно ли $q_{1}$ и $q_{2}$ эквивалентны над $K$ ?

Конечно, для общих квадратичных форм ответ отрицателен, но результаты из раздела 3 показывают, что для этих специальных форм в определенных ситуациях ответ может быть утвердительным.

Теперь мы перейдем к геометрическим задачам о локально симметрических пространствах с соизмеримыми длинами (замкнутых) геодезических, которые привели нас к вопросу $(\dagger)$. Один из подходов к изучению римановых многообразий состоит в том, что с точностью до изометрии или соизмеримости многообразие $M$ должно в значительной степени определяться своим спектром длин $L(M)$, т. е. набором длин всех замкнутых геодезических. В самом простом случае, когда $M_{1}$ и $M_{2}$ являются двумерными евклидовыми сферами, замкнутые геодезические - это большие окружности, и очевидно, что они имеют одинаковые длины тогда и только тогда, когда $M_{1}$ и $M_{2}$ изометричны. Кроме того, применяя формулу следа, можно связать этот подход с задачей о том, когда два 
изоспектральных римановых многообразия (т. е. многообразия, у которых спектры операторов Лапласа-Бельтрами совпадают) изометричны; неформально, это наиболее ярко выражено в вопросе Марка Каца [24] "Можно ли услышать форму барабана?"

Чтобы сделать наши рассуждения более конкретными и в то же время выявить связи с $(\dagger)$, рассмотрим, что происходит с римановыми поверхностями рода $>1$ (детальное обсуждение этих вопросов для общих локально симметрических пространств можно найти в [36]). Пусть

$$
\mathbb{H}=\{x+i y \in \mathbb{C} \mid y>0\}
$$

- верхняя полуплоскость со стандартной гиперболической метрикой $d s^{2}=$ $y^{-2}\left(d x^{2}+d y^{2}\right)$, снабженная обычным изометрическим действием группы $\mathrm{SL}_{2}(\mathbb{R})$ при помощи дробно-линейных преобразований. Пусть $\pi: \mathrm{SL}_{2}(\mathbb{R}) \rightarrow \mathrm{PSL}_{2}(\mathbb{R})-$ каноническая проекция. Напомним, что любая компактная риманова поверхность $M$ рода $>1$ может быть представлена как фактор $\mathbb{H} / \Gamma$, где $\Gamma \subset \mathrm{SL}_{2}(\mathbb{R})-$ дискретная подгруппа, содержащая $\{ \pm 1\}$ и такая, что образ $\pi(\Gamma)$ не имеет кручения (см., например, [17; теорема 27.12]). Хорошо известно, что замкнутые геодезические в $M$ соответствуют нетривиальным полупростым элементам $\gamma \in \Gamma$. Кроме того, поскольку группа $\pi(\Gamma)$ дискретна и не имеет кручения, любой полупростой элемент $\gamma \in \Gamma, \gamma \neq \pm 1$, автоматически является гиперболическим. Следовательно, $\pm \gamma$ сопряжен матрице вида

$$
\left(\begin{array}{cc}
t_{\gamma} & 0 \\
0 & t_{\gamma}^{-1}
\end{array}\right),
$$

где $t_{\gamma}$ - вещественное число, большее 1 . Тогда длина соответствующей замкнутой геодезической $c_{\gamma}$ в $M$ может быть вычислена по формуле

$$
\ell\left(c_{\gamma}\right)=\frac{2}{n_{\gamma}} \cdot \log t_{\gamma},
$$

где $n_{\gamma} \in \mathbb{Z}$ - индекс геодезической $\gamma$ (см. более подробно [36; §8]). Заметим, что, как следует из (1),

$$
\mathbb{Q} \cdot L(M)=\mathbb{Q} \cdot\left\{\log \left|t_{\gamma}\right| \mid \gamma \in \Gamma \backslash\{ \pm 1\}-\text { полупростой элемент }\right\}
$$

$($ множество $\mathbb{Q} \cdot L(M)$ иногда называют рациональным спектром длин поверхности $M)$. Для того чтобы проанализировать эту геометрическую ситуацию используя алгебраические и теоретико-числовые методы, рассматривается алгебра

$$
D=\mathbb{Q}\left[\Gamma^{(2)}\right] \subset M_{2}(\mathbb{R}),
$$

где $\Gamma^{(2)} \subset \Gamma-$ подгруппа, порожденная квадратами. Оказывается, что $D-$ алгебра кватернионов с центром $K$, где $K$ - поле следов группы $\Gamma$, т. е. поле, порожденное над $\mathbb{Q}$ следами $\operatorname{Tr}(\gamma)$ для всех $\gamma \in \Gamma^{(2)}$. Более того, нецентральный полупростой элемент $\gamma \in \Gamma^{(2)}$ определяет максимальную коммутативную 
этальную подалгебру $K[\gamma]$ (см. $[31 ; \S 3.2])$. Следует отметить, что алгебра $D$ является важным инвариантом группы $\Gamma$; в частности, если $\Gamma$ - арифметическая группа, то $D$ - это в точности алгебра, участвующая в ее описании.

Пусть теперь $M_{1}=\mathbb{H} / \Gamma_{1}$ и $M_{2}=\mathbb{H} / \Gamma_{2}$ - две (компактные) римановы поверхности с соответствующими алгебрами кватернионов $D_{i}=\mathbb{Q}\left[\Gamma_{i}^{(2)}\right]$ и полями следов $K_{i}=Z\left(D_{i}\right)$, где $i=1,2$. Предположим, что $M_{1}$ и $M_{2}$ имеют соизмеримъье длины геодезических (условие (L-C)), т. е.

$$
\mathbb{Q} \cdot L\left(M_{1}\right)=\mathbb{Q} \cdot L\left(M_{2}\right) .
$$

Это влечет, что $K_{1}=K_{2}=: K$ (см. [36; теорема 2]). ${ }^{1}$ Кроме того, как следует из $(1)$, для любого нетривиального полупростого элемента $\gamma_{1} \in \Gamma_{1}^{(2)}$ существует такой нетривиальный полупростой элемент $\gamma_{2} \in \Gamma_{2}^{(2)}$, что

$$
t_{\gamma_{1}}^{m}=t_{\gamma_{2}}^{n}
$$

для некоторых целых чисел $m, n \geqslant 1$. Следовательно, матрицы $\gamma_{1}^{m}$ и $\gamma_{2}^{n}$ сопряжены в $\mathrm{GL}_{2}(\mathbb{R})$, и мы имеем изоморфизм соответствующих этальных алгебр:

$$
K\left[\gamma_{1}\right]=K\left[\gamma_{1}^{m}\right] \simeq K\left[\gamma_{2}^{n}\right]=K\left[\gamma_{2}\right]
$$

Таким образом, геометрическое условие (L-C) преобразуется в алгебраическое условие: $D_{1}$ и $D_{2}$ имеют одинаковые классы изоморфизма максимальных эталъных подалгебр, пересекающих $\Gamma_{1}^{(2)} u \Gamma_{2}^{(2)}$ соответственно.

На самом деле хотелось бы доказать, что из (L-C) следует, что $M_{1}$ и $M_{2}$ соизмеримы (т.е. имеют общее конечнолистное накрытие или, эквивалентно, с точностью до сопряженности подгруппы $\Gamma_{1}$ и $\Gamma_{2}$ соизмеримы в $\left.\mathrm{GL}_{2}(\mathbb{R})\right)$. Если это так, то мы имеем $D_{1} \simeq D_{2}$ (см. [31; следствие 3.3.5]). Итак, проблема соизмеримости римановых поверхностей приводит к следующему вопросу об алгебрах кватернионов.

Пусть $D_{1}$ и $D_{2}$ - две центральные алгебры кватернионов над полем $K$, и пусть $\Gamma_{i} \subset \mathrm{SL}\left(1, D_{i}\right), i=1,2,-$ плотные по Зарискому подгруппы с полем следов $K$. Допустим, что $D_{1}$ и $D_{2}$ имеют одинаковые классы изоморфизма максимальных этальных подалгебр, пересекающих $\Gamma_{1} u \Gamma_{2}$ соответственно. Являются ли $D_{1}$ и $D_{2}$ изоморфными?

Это более тонкий вариант нашего исходного вопроса $(\dagger)$. Если $K$ - числовое поле, то, как мы уже упоминали, два тела кватернионов, имеющих одинаковые максимальные подполя, изоморфны (см. более подробно в разделе 3). Этот факт был использован А. Ридом [41] для того, чтобы показать, что любые две арифметически определенные римановы поверхности с одинаковым спектром длин геодезических (т. е. $M_{1}$ и $M_{2}$ такие, что $L\left(M_{1}\right)=L\left(M_{2}\right)$ ) соизмеримы. Общий случай, скорее всего, зависит от ответов на вопросы, сформулированные выше.

\footnotetext{
${ }^{1}$ На самом деле равенство полей следов доказывается исходя из предположения о слабой соизмеримости $\Gamma_{1}$ и $\Gamma_{2}$, которое в свою очередь связано с соизмеримостью длин замкнутых геодезических соответствующих римановых поверхностей.
} 
Мы хотели бы завершить наше обсуждение двумя замечаниями. Во-первых, надо отметить, что если подгруппа Г неарифметична, то она не определяется однозначно соответствующей алгеброй кватернионов (в действительности, можно построить бесконечное семейство несоизмеримых кокомпактных решеток, имеющих одну и ту же кватернионную алгебру, см. [48]), поэтому из утвердительного ответа на наш вопрос об алгебрах не будет непосредственно следовать результат для римановых поверхностей. Во-вторых, помимо точного (количественного) варианта сформулированного выше вопроса можно рассмотреть его качественную версию: всегда ли множество классов изоморфизма алгебр с делением, имеющих одинаковые максимальные подполя, конечно? Отметим, что подобные результаты о конечности действительно имеются даже для неарифметических римановых поверхностей. Например, известно, что каждый класс изоспектральных компактных римановых поверхностей распадается в объединение конечного числа классов изометрических поверхностей. Напомним, что, согласно известной гипотезе, каждый класс изоспектральных поверхностей должен состоять из одного класса соизмеримости.

\section{3. Род алгебры с делением}

В этом разделе мы дадим обзор имеющихся результатов по вопросам 1 и 2 из раздела 1 о роде алгебры с делением.

Начнем с нескольких замечаний к вопросу 1 . Во-первых, отметим, что род $D$ может быть тривиальным только в том случае, когда класс $[D]$ имеет порядок 2 в группе Брауэра. Действительно, противоположная алгебра $D^{\text {ор }}$ имеет те же максимальные подполя, что и $D$. Из этого следует, что если $|\operatorname{gen}(D)|=1$, то $D \simeq D^{\text {ор }}$ (что эквивалентно тому, что $[D]$ имеет порядок 2 ). В то же время, как показывает пример кубической алгебры над числовым полем, приведенный в разделе 1 , род заведомо может быть больше, чем $\left\{[D],\left[D^{\text {op }}\right]\right\}$. Другой такой пример можно построить следующим образом. Пусть $\Delta_{1}$ и $\Delta_{2}$ - центральные алгебры с делением над полем $K$, имеющие взаимно простые нечетные степени. Рассмотрим алгебры с делением

$$
D_{1}=\Delta_{1} \otimes_{K} \Delta_{2} \quad \text { и } \quad D_{2}=\Delta_{1} \otimes_{K} \Delta_{2}^{\mathrm{op}} .
$$

Тогда $D_{2}$ не изоморфна ни $D_{1}$, ни $D_{1}^{\text {op }}$, но $D_{1}$ и $D_{2}$ имеют одни и те же поля разложения. Действительно, поскольку степени $\Delta_{1}$ и $\Delta_{2}$ взаимно просты, расширение расщепляет $D_{1}$ тогда и только тогда, когда оно расщепляет $\Delta_{1}$ и $\Delta_{2}$; тогда оно также расщепляет $\Delta_{2}^{\text {op }}$ и, следовательно, $D_{2}$ (и обратно). Итак, $D_{1}$ и $D_{2}$ имеют одинаковые максимальные подполя, и поэтому $\left[D_{2}\right] \in \operatorname{gen}\left(D_{1}\right)$. Обратим внимание, что этот пример вытекает из следующего общего факта: если $D$ - центральная алгебра с делением степени $n$ над полем $K$, то для любого целого $m$, взаимно простого с $n$, класс $\left[D^{\otimes m}\right]=m[D] \in \operatorname{Br}(K)$ представим центральной алгеброй с делением $D_{m}$ степени $n$, которая имеет те же максимальные подполя, что и $D$ (см. [40; лемма 3.6]). 
Как мы уже отмечали, теорема Альберта-Брауэра-Хассе-Нётер (ABHN) позволяет полностью ответить на вопросы 1 и 2 в случае, когда $K$ - числовое поле. Точное утверждение выглядит следующим образом.

ПреДЛОЖеНИЕ 3.1. Предположим, что $K$ - числовое поле $u D$ - конечномерная иентральная алгебра с делением над $K$.

(а) Если $[D] \in \operatorname{Br}(K)$ имеет порядок 2 (в этом случае $D$ - алгебра кватернионов), то $|\operatorname{gen}(D)|=1$.

(b) Род $\operatorname{gen}(D)$ является конечным множеством для всех D.

Напомним, что из $(\mathrm{ABHN})$ мы имеем инъективный гомоморфизм

$$
0 \rightarrow \operatorname{Br}(K) \rightarrow \bigoplus_{v \in V^{K}} \operatorname{Br}\left(K_{v}\right),
$$

где для каждого $v \in V^{K}$

$$
\operatorname{Br}(K) \rightarrow \operatorname{Br}\left(K_{v}\right), \quad[D] \mapsto\left[D \otimes_{K} K_{v}\right]
$$

- естественное отображение. Будем говорить, что конечномерная центральная алгебра с делением $D$ над числовым полем $K$ (или ее класс $[D] \in \operatorname{Br}(K))$ неразветвлена в $v \in V^{K}$, если ее образ в $\operatorname{Br}\left(K_{v}\right)$ тривиален, и разветвлена в противном случае. Заметим, что это определение согласуется с понятием ветвления над произвольными полями (см. раздел 4 и, в частности, пример 4.1). Конечное множество нормирований, в которых $D$ разветвлено, будем обозначать через $R(D)$.

НАБРОСОК ДОКАЗАТЕЛЬСТВА ПРЕДЛОЖЕНИЯ 3.1. (а) СНачала отметим, что алгебра с делением $D$ над $K$ экспоненты 2 является алгеброй кватернионов в силу того, что над числовыми полями степень алгебры с делением и ее экспонента совпадают, что следует из теоремы Грюнвальда-Ванга (см., например, [33; гл. VIII, §2]). Далее, рассмотрим 2-кручение в $(\mathrm{ABHN})$ :

$$
0 \rightarrow{ }_{2} \operatorname{Br}(K) \rightarrow \bigoplus_{v \in V^{K}}{ }_{2} \operatorname{Br}\left(K_{v}\right)
$$

Так как ${ }_{2} \operatorname{Br}\left(K_{v}\right)$ изоморфна либо $\mathbb{Z} / 2 \mathbb{Z}$, либо 0 для всех $v \in V^{K}$, мы видим, что алгебра $D$ степени 2 над $K$ однозначно, с точностью до изоморфизма, определяется своим ветвлением $R(D)$. Следовательно, чтобы доказать равенство $|\operatorname{gen}(D)|=1$, достаточно показать, что если две алгебры кватернионов с делением $D_{1}$ и $D_{2}$ имеют общие максимальные подполя, то $R\left(D_{1}\right)=R\left(D_{2}\right)$. Это легко следует из слабой аппроксимации и хорошо известного критерия, который утверждает, что для $d \in K^{\times} \backslash\left(K^{\times}\right)^{2}$

$$
L=K(\sqrt{d}) \text { обладает вложением в } D \Leftrightarrow d \notin\left(K_{v}^{\times}\right)^{2} \text { для всех } v \in R(D)
$$

(см. [34; § 18.4, следствие b]). Действительно, предположим, что существует $v_{0} \in R\left(D_{1}\right) \backslash R\left(D_{2}\right)$. Используя открытость $\left(K_{v}^{\times}\right)^{2} \subset K_{v}^{\times}$и слабую аппрокси- 
мацию, можно найти такой $d \in K^{\times} \backslash\left(K^{\times}\right)^{2}$, что

$$
d \in\left(K_{v_{0}}^{\times}\right)^{2}, \quad \text { но } \quad d \notin\left(K_{v}^{\times}\right)^{2} \text { для всех } v \in R\left(D_{2}\right) .
$$

Тогда, согласно (2), квадратичное расширение $L=K(\sqrt{d})$ обладает вложением в $D_{2}$, но не может быть вложено в $D_{1}-$ противоречие.

(b) Пусть $D$ - алгебра с делением над $K$ степени $n>2$. Рассмотрим $n$-кручение в (ABHN):

$$
0 \rightarrow{ }_{n} \operatorname{Br}(K) \rightarrow \bigoplus_{v \in V^{K}}{ }_{n} \operatorname{Br}\left(K_{v}\right)
$$

Для того чтобы установить конечность gen $(D)$, сначала отметим, что из $D^{\prime} \in$ $\operatorname{gen}(D)$ следует равенство $R\left(D^{\prime}\right)=R(D)$ (см. [34; 18.4 , следствие b]; на самом деле это верно не только над числовыми полями - см. лемму 4.2 ниже). Так как ${ }_{n} \operatorname{Br}\left(K_{v}\right)$ - это $\mathbb{Z} / n \mathbb{Z}, \mathbb{Z} / 2 \mathbb{Z}$ или 0 для всех $v \in V^{K}$ (см. пример 4.1 , (а) ниже), мы имеем

$$
|\operatorname{gen}(D)| \leqslant\left|\bigoplus_{v \in R(D)}{ }_{n} \operatorname{Br}\left(K_{v}\right)\right| \leqslant n^{r},
$$

где $r=|R(D)|$.

Для иллюстрации рассуждений, приведенных в доказательстве утверждения (а), рассмотрим следующий пример.

ПРИМЕР 3.2. Пусть

$$
D_{1}=\left(\frac{-1,3}{\mathbb{Q}}\right) \quad \text { и } \quad D_{2}=\left(\frac{-1,7}{\mathbb{Q}}\right) .
$$

Можно проверить, что $R\left(D_{1}\right)=\{2,3\}$ и $R\left(D_{2}\right)=\{2,7\}$, следовательно, $D_{1}$ и $D_{2}$ - неизоморфные тела кватернионов над $\mathbb{Q}$. Очевидно, что $10 \in\left(\mathbb{Q}_{3}^{\times}\right)^{2}$, но $10 \notin\left(\mathbb{Q}_{2}^{\times}\right)^{2},\left(\mathbb{Q}_{7}^{\times}\right)^{2}$. Таким образом, согласно $(2)$ поле $L=\mathbb{Q}(\sqrt{10})$ может быть вложено в $D_{2}$, но не в $D_{1}$. Другими словами, $D_{1}$ и $D_{2}$ различаются своими квадратичными подполями. Мы хотели бы отметить, что в недавнем препринте [30] найдена эффективная оценка дискриминанта квадратичного поля, которое различает два данных несовпадающих тела кватернионов [30; теорема 1.3].

Теперь естественно спросить, переносятся ли (и в какой мере) полученные результаты про род на более общие поля. А именно, можно ли ожидать, что род всегда тривиален для алгебры кватернионов с делением и конечен для любой конечномерной алгебры с делением над произвольным полем $K$ ? Оказывается, что в обоих случаях ответ отрицателен. Конструкция алгебр кватернионов с нетривиальным родом над некоторыми очень большими полями была предложена М. Ростом, М. Шахером, А. Вадсвортом и др. За детальным описанием конструкции мы отсылаем читателя к $[20 ; \S 2]$, а здесь только наметим основные идеи. 
Пусть $D_{1}$ и $D_{2}$ - две неизоморфные алгебры кватернионов с делением над полем $k$ характеристики $\neq 2$, содержащие фиксированное квадратичное расширение $k$. Например, можно взять $k=\mathbb{Q}$ и рассмотреть алгебры кватернионов $D_{1}$ и $D_{2}$ из примера 3.2. Если $D_{1}$ и $D_{2}$ уже имеют общие квадратичные подполя, то все доказано. Если нет, то существует квадратичное расширение $k(\sqrt{d})$, которое может быть вложено в $D_{1}$, но не в $D_{2}$. Применяя стандартные результаты о квадратичных формах к норменным формам $D_{1}$ и $D_{2}$, можно показать, что существует такое расширение $k^{(1)}$ поля $k$ (являющееся полем рациональных функций на некоторой $k$-определенной квадрике), что

- $D_{1} \otimes_{k} k^{(1)}$ и $D_{2} \otimes_{k} k^{(1)}$ - неизоморфные алгебры с делением над $k^{(1)}$, но

- $k^{(1)}(\sqrt{d})$ обладает вложением в $D_{2} \otimes_{k} k^{(1)}$.

С оставшимися подполями мы разбираемся последовательно, применяя ту же процедуру к алгебрам, полученным из $D_{1}$ и $D_{2}$ путем расширения скаляров, выполненного на предыдущем шаге. Этот процесс порождает возрастающую цепочку полей

$$
k^{(1)} \subset k^{(2)} \subset k^{(3)} \subset \cdots,
$$

и мы берем в качестве $K$ объединение (прямой предел) этой цепочки. Тогда $D_{1} \otimes_{k} K$ и $D_{2} \otimes_{k} K$ оказываются неизоморфными телами кватернионов над $K$ с одинаковыми максимальными подполями; в частности, $\left|\operatorname{gen}\left(D_{1} \otimes_{k} K\right)\right|>1$. Заметим, что полученное поле $K$ имеет бесконечную степень трансцендентности над $k$ и, в частности, является бесконечно порожденным. Более того, применяя некоторую модификацию данной конструкции (см. [32]) к бесконечному набору попарно неизоморфных тел кватернионов $D_{1}, D_{2}, D_{3}, \ldots$ над полем $k$ характеристики $\neq 2$, содержащих фиксированное квадратичное расширение (например, возьмем $k=\mathbb{Q}$ и рассмотрим семейство алгебр вида $\left(\frac{-1, p}{\mathbb{Q}}\right)$, где $p$ - простое число $\equiv 3(\bmod 4))$, можно построить такое бесконечно порожденное расширение $K / k$, что алгебры $D_{i} \otimes_{k} K$ остаются попарно неизоморфными телами кватернионов, причем любые две из них имеют одинаковые квадратичные подполя. В частности, это дает пример тела кватернионов, имеющего бесконечный род. Совсем недавно аналогичный подход был использован в [47], чтобы показать, что для любого простого $p$ существуют такие поле $K$ и центральная алгебра с делением $D$ над $K$ степени $p$, что gen $(D)$ бесконечен.

Таким образом, в то время как над числовыми и, более общо, глобальными полями вопросы 1 и 2 получают исчерпывающий ответ, над произвольными полями они оказываются весьма нетривиальными. На самом деле до недавнего времени практически ничего не было известно о ситуации над полями, отличными от глобальных. Как мы уже упоминали в разделе 2 , тривиальность рода для тел кватернионов $D$ над числовыми полями имеет следствия для арифметически определенных римановых поверхностей. В связи с исследованием локально симметрических пространств с соизмеримыми длинами геодезических [36] Г. Прасад и второй из авторов настоящей статьи задали вопрос, всегда 
ли $|\operatorname{gen}(D)|=1$ для любого центрального тела кватернионов над $K=\mathbb{Q}(x) .^{2}$ Утвердительный ответ был дан Д. Солтманом, а позже в совместной работе Д. Солтман и С. Гарибальди показали, что любое тело кватернионов над $k(x)$, где $k$ - произвольное числовое поле, имеет тривиальный род (на самом деле ими был рассмотрен более общий случай так называемых прозрачных (transparent) полей характеристики $\neq 2$ - см. [20]). Мотивированные этим результатом, мы показали в [40], что тривиальность рода произвольных тел кватернионов над данным полем $k$ характеристики $\neq 2$ является свойством, которое стабильно при чисто трансцендентных расширениях. Впоследствии мы установили аналогичную теорему стабилъности для произвольных алгебр экспоненты 2. Точные формулировки выглядят следующим образом.

Теорема 3.3 (см. [40; теорема A] и [5; теорема 3.5]). Пусть $k$ - поле характеристики $\neq 2$.

(a) Если $|\operatorname{gen}(D)|=1$ для любого тела кватернионов $D$ над $k$, то

$$
\left|\operatorname{gen}\left(D^{\prime}\right)\right|=1
$$

для любого тела кватернионов $D^{\prime}$ над полем рациональных функиий $k(x)$.

(b) $E c л и|\operatorname{gen}(D)|=1$ для любой центральной $k$-алгебры с делением экспоненты 2, то это свойство справедливо для любой централъной алгебры с делением экспоненты 2 над $k(x)$.

В качестве непосредственного следствия мы получаем такое утверждение.

СЛЕДСТВИЕ 3.4. Если $k$ - числовое или конечное поле характеристики $\neq 2$ u $K=k\left(x_{1}, \ldots, x_{r}\right)$ - его чисто трансцендентное расширение, то для любой иентральной $K$-алгебры с делением $D$ экспоненты 2 выполнено равенство $|\operatorname{gen}(D)|=1$.

ЗАмЕчАниЕ 3.5. В [26] теорема стабильности была обобщена на поля функций многообразий Севери-Брауэра алгебр нечетной степени.

Обратимся теперь к вопросу 2. Выше мы видели, что невозможно рассчитывать на получение результата о конечности для рода над произвольными полями. Тем не менее мы доказали следующее утверждение о конечности над конечно порожденными полями.

Теорема 3.6 [4; теорема 3]. Пусть $K$ - конечно порожденное поле. Если $D$ - иентральная $K$-алгебра с делением степени, взаимно простой $c$ char $K$, то $\operatorname{gen}(D)$ - конечное множество.

ЗАмЕчАниЕ 3.7. (а) Остается открытым вопрос, существуют ли тела кватернионов над конечно порожденными полями с нетривиальным родом.

(b) В [27] Д. Крашен и К. Маккини изучали алгебры с делением, имеющие одинаковые конечномерные поля разложений (соответствующее понятие рода обсуждается в разделе 5).

\footnotetext{
${ }^{2}$ Хотя вопросы такого рода обсуждались по крайней мере начиная с работы Ш. Амицура [2] (см. раздел 1), тем не менее, насколько нам известно, это было первой "официальной" постановкой; сам термин род был введен позже.
} 


\section{4. Ветвление алгебр с делением}

В этом разделе мы опишем некоторые идеи, задействованные в доказательствах теорем 3.3 и 3.6. По большому счету, наши рассуждения во многом опираются на те же общие соображения, которые мы уже обсуждали в случае числовых полей. Точнее, несмотря на отсутствие прямого аналога (ABHN) для произвольных (конечно порожденных) полей, анализ ветвлений алгебр с делением опять играет ключевую роль.

Для начала напомним стандартные факты, которые используются в анализе ветвлений алгебр с делением. Пусть $K$ - поле с дискретным нормированием $v$. Обозначим через $\mathscr{G}^{(v)}=\operatorname{Gal}\left(\bar{K}_{v}^{\mathrm{sep}} / \bar{K}_{v}\right)$ абсолютную группу Галуа поля вычетов $\bar{K}_{v}$ пополнения $K_{v}$ и зафиксируем целое $n>1$. Если $n$ и $\operatorname{char} \bar{K}_{v}$ взаимно просты или $\bar{K}_{v}$ совершенно, то существует отображение вычета

$$
r_{v}:{ }_{n} \operatorname{Br}\left(K_{v}\right) \rightarrow \operatorname{Hom}\left(\mathscr{G}^{(v)}, \mathbb{Z} / n \mathbb{Z}\right),
$$

где группа справа является группой непрерьвных характеров группы $\mathscr{G}(v)$ по модулю $n$ (см. $[43 ; \S 10])$. Кроме того, известно, что $r_{v}$ сюръективно. Алгебра с делением $D$ над $K_{v}$ экспоненты $n$ (или соответствующий класс $[D] \in{ }_{n} \operatorname{Br}\left(K_{v}\right)$ ) называется неразветвленной, если $r_{v}([D])=0$, а неразветвленная группа Брауэра поля $K_{v}$ определяется как

$$
{ }_{n} \operatorname{Br}\left(K_{v}\right)_{\{v\}}=\operatorname{ker} r_{v} .
$$

Таким образом, по построению, мы имеем точную последовательность

$$
0 \rightarrow{ }_{n} \operatorname{Br}\left(K_{v}\right)_{\{v\}} \rightarrow{ }_{n} \operatorname{Br}\left(K_{v}\right) \stackrel{r_{v}}{\longrightarrow} \operatorname{Hom}\left(\mathscr{G}^{(v)}, \mathbb{Z} / n \mathbb{Z}\right) \rightarrow 0 .
$$

Можно показать, что существует изоморфизм ${ }_{n} \operatorname{Br}\left(K_{v}\right)_{\{v\}} \simeq{ }_{n} \operatorname{Br}\left(\bar{K}_{v}\right)$ и, более того, последняя группа естественно отождествляется с ${ }_{n} \operatorname{Br}\left(\mathscr{O}_{v}\right)$, где $\mathscr{O}_{v}-$ кольцо нормирования $K_{v}$ (см. [51; теорема 3.2 и последующие рассуждения]). Другими словами, неразветвленные относительно $v$ алгебры - это в точности те, которые возникают из алгебр Азумая над $\mathscr{O}_{v}$.

Далее, используя композицию отображения (4) и естественного гомоморфизма ${ }_{n} \operatorname{Br}(K) \rightarrow{ }_{n} \operatorname{Br}\left(K_{v}\right)$, получаем отображение вычета

$$
\rho_{v}:{ }_{n} \operatorname{Br}(K) \rightarrow \operatorname{Hom}\left(\mathscr{G}^{(v)}, \mathbb{Z} / n \mathbb{Z}\right),
$$

и снова мы говорим, что алгебра с делением $D$ над $K$ неразветвлена относительно $v$, если $\rho_{v}([D])=0$. В случае полей, отличных от локальных, для получения информации о ${ }_{n} \operatorname{Br}(K)$, как правило, используется не индивидуальное нормирование $K$, а подходящий набор нормирований. Для этой цели удобно дать следующее определение. Зафиксируем целое число $n>1$ и обозначим через $V$ множество таких дискретных нормирований поля $K$, что отображение вычета $\rho_{v}$ определено для всех $v \in V$. Тогда $n$-кручение неразветвленной группь Брауэра относительно $V$ определяется следующим образом:

$$
{ }_{n} \operatorname{Br}(K)_{V}=\bigcap_{v \in V} \operatorname{ker} \rho_{v} .
$$


ПримеР 4.1. (а) Пусть $p$ - простое число, $K=\mathbb{Q}_{p}$ и $v=v_{p}$ - соответствующее $p$-адическое нормирование. Тогда, поскольку поле $\mathbb{F}_{p}$ из $p$ элементов совершенно и $\operatorname{Br}\left(\mathbb{F}_{p}\right)=\{0\}$, из (5) следует, что для любого $n>1$ существует изоморфизм

$$
{ }_{n} \operatorname{Br}\left(\mathbb{Q}_{p}\right) \stackrel{r_{v}}{\simeq} \operatorname{Hom}\left(\operatorname{Gal}\left(\overline{\mathbb{F}}_{p} / \mathbb{F}_{p}\right), \mathbb{Z} / n \mathbb{Z}\right)=\operatorname{Hom}_{\text {cont }}(\widehat{\mathbb{Z}}, \mathbb{Z} / n \mathbb{Z}) \simeq \mathbb{Z} / n \mathbb{Z} .
$$

Переходя к пределу по всем $n$, мы получаем изоморфизм $\operatorname{Br}\left(\mathbb{Q}_{p}\right) \simeq \mathbb{Q} / \mathbb{Z}$, который совпадает с инвариантом из локальной теории полей классов. Это описание группы Брауэра распространяется на любое конечное расширение $\mathbb{Q}_{p}$. Важным моментом здесь является то, что только тривиальная алгебра над таким полем является неразветвленной (относительно естественного нормирования).

(b) Пусть $K$ - числовое поле. Из (а) следует, что конечномерная центральная алгебра с делением $D$ над $K$ является неразветвленной относительно неархимедова нормирования $v \in V^{K}$ тогда и только тогда, когда ее образ в $\operatorname{Br}\left(K_{v}\right)$ тривиален (что согласуется с определением, которое мы использовали в разделе 3). Используя теперь точность (ABHN), мы видим, что если $S \subset V^{K}$ конечное множество, содержащее $V_{\infty}^{K}$, то для $V=V^{K} \backslash S$ и любого целого $n>1$ неразветвленная группа Брауэра ${ }_{n} \operatorname{Br}(K)_{V}$ конечна.

(c) Пусть $C$ - гладкая связная проективная кривая над полем $k$ и $K=k(C)-$ поле функций на $C$. Каждой замкнутой точке $P \in C$ на $K$ отвечает дискретное нормирование $v_{P}$ поля $K$. Множество дискретных нормирований

$$
V_{0}=\left\{v_{P} \mid P \in C-\text { замкнутая точка }\right\}
$$

обычно называют множеством геометрических плейсов $K$ (отметим, что это в точности дискретные нормирования $K$, которые тривиальны на $k$ ). Если $n>1$ - целое, взаимно простое с $\operatorname{char} k$, то для каждого $v_{P} \in V_{0}$ имеется отображение вычета, ассоциированное с точкой $P$ :

$$
\rho_{P}=\rho_{v_{P}}:{ }_{n} \operatorname{Br}(K) \rightarrow H^{1}\left(G_{P}, \mathbb{Z} / n \mathbb{Z}\right),
$$

где $G_{P}$ - абсолютная группа Галуа поля вычетов точки $P$. В этом случае соответствующую неразветвленную группу Брауэра ${ }_{n} \operatorname{Br}(K)_{V_{0}}$, следуя традиции, будем обозначать через ${ }_{n} \operatorname{Br}(K)$ ur. Кроме того, если $k$ - совершенное поле и $C$ геометрически связна, то определенное выше отображение вычета $\rho_{P}$ можно продолжить до отображения $\operatorname{Br}(K) \rightarrow H^{1}\left(G_{P}, \mathbb{Q} / \mathbb{Z}\right)$, определенного на всей группе Брауэра $\operatorname{Br}(K)$. В этом случае мы можем рассмотреть отображение

$$
\operatorname{Br}(K) \stackrel{\oplus \rho_{P}}{\longrightarrow} \bigoplus_{P \in C_{0}} H^{1}\left(G_{P}, \mathbb{Q} / \mathbb{Z}\right),
$$

где $C_{0}-$ множество замкнутых точек кривой $C$. Известно, что его ядро

$$
\operatorname{ker}\left(\bigoplus \rho_{P}\right)=: \operatorname{Br}(K)_{\mathrm{ur}}
$$

совпадает с группой Брауэра $\operatorname{Br}(C)$ кривой $C$, определенной либо в терминах алгебр Азумая, либо в терминах этальных когомологий (см. [21; §6.4] и [29]). 
Таким образом, группа ${ }_{n} \operatorname{Br}(K)$ ur совпадает с подгруппой $n$-кручения группы $\operatorname{Br}(C)$.

Возвращаясь к общей ситуации, мы сейчас сформулируем результат, который описывает ветвление алгебр с делением из одного рода. Пусть $K$ - поле с дискретным нормированием $v$, и пусть $n>1$ - целое число, взаимно простое с характеристикой поля вычетов $\bar{K}_{v}$. Имеет место следующее утверждение.

Лемма 4.2. Пусть $D$ и $D^{\prime}$ - центральные $K$-алгебры с делением такие, ymo

$$
[D] \in{ }_{n} \operatorname{Br}(K) \quad u \quad\left[D^{\prime}\right] \in \operatorname{gen}(D) \cap{ }_{n} \operatorname{Br}(K) .
$$

Обозначим через $\chi_{v}$ u $\chi_{v}^{\prime}$ образы $D$ и $D^{\prime}$ в $\operatorname{Hom}\left(\mathscr{G}^{(v)}, \mathbb{Z} / n \mathbb{Z}\right)$ при отображении вычета $\rho_{v}$. Тогда

$$
\operatorname{ker} \chi_{v}=\operatorname{ker} \chi_{v}^{\prime} .
$$

В частности, $D$ неразветвлено относительно $v$ в том и только том случае, когда этим свойством обладает $D^{\prime}$.

Прежде чем доказывать лемму, напомним, что если поле $\mathscr{K}$ является полным относительно дискретного нормирования $v$ и $\mathscr{D}$ - конечномерная центральная алгебра с делением над $\mathscr{K}$, то $v$ можно однозначно продолжить до дискретного нормирования $\widetilde{v}$ тела $\mathscr{D}$ (см. [45; гл. ХII, §2], [51]). Далее, соответствующее кольцо нормирования $\mathscr{O}_{\mathscr{D}}$ имеет единственный максимальный 2-сторонний идеал $\mathfrak{P}_{\mathscr{D}}$ (идеал нормирования), и факторкольцо $\overline{\mathscr{D}}=\mathscr{O}_{\mathscr{D}} / \mathfrak{P}_{\mathscr{D}}$ является конечномерной алгеброй с делением над полем вычетов $\overline{\mathscr{K}}$ (не обязательно центральной), называемой алгеброй вычетов.

ДОКАЗАТЕЛЬСТво (НАБРОСОК). Пусть

$$
D \otimes_{K} K_{v}=M_{\ell}(\mathscr{D}) \quad \text { и } \quad D^{\prime} \otimes_{K} K_{v}=M_{\ell^{\prime}}\left(\mathscr{D}^{\prime}\right),
$$

где $\mathscr{D}$ и $\mathscr{D}^{\prime}$ - центральные алгебры с делением над $K_{v}$. Вначале показывается, что $\ell=\ell^{\prime}$, а $\mathscr{D}$ и $\mathscr{D}^{\prime}$ имеют одинаковые максимальные подполя [40; следствие 2.4]. Затем проверяется, что центры $\mathscr{E}$ и $\mathscr{E}^{\prime}$ алгебр вычетов $\overline{\mathscr{D}}$ и $\overline{\mathscr{D}}^{\prime}$ совпадают [5; лемма 2.3]. Наконец, используя тот факт, что ker $\chi_{v}$ и ker $\chi_{v}^{\prime}$ являются в точности подгруппами группы $\mathscr{G}^{(v)}$, соответствующими $\mathscr{E}$ и $\mathscr{E}^{\prime}$ (см. [51; теорема 3.5]), мы заключаем, что

$$
\operatorname{ker} \chi_{v}=\operatorname{ker} \chi_{v}^{\prime}
$$

как и требовалось.

Теперь мы можем дать набросок доказательства части (а) теоремы 3.3 (см. подробности в доказательстве теоремы А в [40]). Пусть $k$ - такое поле характеристики $\neq 2$, что $|\operatorname{gen}(D)|=1$ для каждой алгебры кватернионов с делением $D$ над $k$. Отождествим $k(x)$ с полем рациональных функций на проективной прямой $\mathbb{P}_{k}^{1}$ и рассмотрим следующий фрагмент точной последовательности Фаддеева (см. [21; следствие 6.4.6]):

$$
0 \rightarrow{ }_{2} \operatorname{Br}(k) \rightarrow{ }_{2} \operatorname{Br}(k(x)) \stackrel{\oplus \rho_{P}}{\longrightarrow} \bigoplus_{P \in\left(\mathbb{P}_{k}^{1}\right)_{0}} H^{1}\left(G_{P}, \mathbb{Z} / 2 \mathbb{Z}\right) .
$$


Предположим, что $D$ и $D^{\prime}$ - алгебры кватернионов с делением над $k(x)$, имеющие общие максимальные подполя. Учитывая, что $[D]$ и $\left[D^{\prime}\right]$ имеют экспоненту 2 в $\operatorname{Br}(k(x))$, мы получаем из леммы 4.2 , что

$$
\rho_{P}([D])=\rho_{P}\left(\left[D^{\prime}\right]\right)
$$

для всех $P \in\left(\mathbb{P}_{k}^{1}\right)_{0}$. Следовательно, из $(7)$ получаем, что

$$
[D]=\left[D^{\prime}\right] \cdot\left[\Delta \otimes_{k} k(x)\right]
$$

для некоторой центральной алгебры кватернионов $\Delta$ над $k$ (см. [40; следствие 4.2]). С помощью процедуры специализации, используя предположение о том, что тела кватернионов над $k$ имеют тривиальный род, показывается, что $[\Delta]=0$, и, значит, $D \simeq D^{\prime}$. Таким образом, $|\operatorname{gen}(D)|=1$, что и требовалось.

Сейчас мы опишем основные шаги доказательства теоремы 3.6, которое также опирается на анализ ветвления. Пусть $K$ - конечно порожденное поле. Зафиксируем целое $n>1$, взаимно простое с char $K$. Рассмотрим множество $V$ дискретных нормирований поля $K$, обладающее следующими двумя свойствами:

(I) для любого $a \in K^{\times}$множество $V(a):=\{v \in V \mid v(a) \neq 0\}$ конечно;

(II) для любого $v \in V$ характеристика поля вычетов $\bar{K}_{v}$ взаимно проста $c$. Заметим, что (II) обеспечивает существование отображения вычета

$$
{ }_{n} \operatorname{Br}(K) \stackrel{\rho_{v}}{\longrightarrow} \operatorname{Hom}\left(\mathscr{G}^{(v)}, \mathbb{Z} / n \mathbb{Z}\right)
$$

для каждого $v \in V$. При рассмотрении случая числовых полей в разделе 3 мы заметили, что множество разветвленных нормирований конечно для любой алгебры с делением $D$, и это, с помощью (ABHN), привело к оценке (3) размера gen $(D)$. Над общими полями условие (I) снова гарантирует конечность множества

$$
R(D)=R(D, V)=\left\{v \in V \mid \rho_{v}([D]) \neq 0\right\}
$$

разветвленных нормирований для любой алгебры с делением $D$ степени $n$ над $K$ (см. несколько более общее утверждение в [5; предложение 2.1]). Для получения аналога оценки (3) мы рассуждаем следующим образом, используя лемму 4.2. Предположим, что $D$ - центральная алгебра с делением над $K$ степени $n$ и $\left[D^{\prime}\right] \in \operatorname{gen}(D)$. Для $v \in V$ положим

$$
\chi_{v}=\rho_{v}([D]) \quad \text { и } \quad \chi_{v}^{\prime}=\rho_{v}\left(\left[D^{\prime}\right]\right) ;
$$

напомним, что по лемме 4.2 имеем $\operatorname{ker} \chi_{v}=\operatorname{ker} \chi_{v}^{\prime}$. Отметим, что если характер $\chi_{v}$ имеет порядок $m \mid n$, то любой характер $\chi_{v}^{\prime}$ группы $\mathscr{G}(v)$ с тем же ядром можно рассматривать как точный характер циклической группы $\mathscr{G}^{(v)} / \operatorname{ker} \chi_{v}$ порядка $m$. Следовательно, существует $\varphi(m)$ возможностей для $\chi^{\prime}$, и поэтому

$$
\left|\rho_{v}(\operatorname{gen}(D))\right| \leqslant \varphi(m) \leqslant \varphi(n)
$$

для любого $v \in V$ (поскольку $m$ делит $n)$, и

$$
\rho_{v}(\operatorname{gen}(D))=\{1\},
$$


если $\rho_{v}([D])=1$. Пусть $\rho=\left(\rho_{v}\right)_{v \in V}$ - прямая сумма отображений вычета для всех $v \in V$. Тогда

$$
|\rho(\operatorname{gen}(D))| \leqslant \varphi(n)^{r},
$$

где $r=|R(D)|$. Следовательно, если $\operatorname{ker} \rho={ }_{n} \operatorname{Br}(K)_{V}$ конечно, мы получим оценку

$$
|\operatorname{gen}(D)| \leqslant \varphi(n)^{r} \cdot\left|{ }_{n} \operatorname{Br}(K)_{V}\right| .
$$

Таким образом, доказательство теоремы 3.6 сводится к установлению конечности неразветвленной группы Брауэра относительно подходящего множества дискретных нормирований, что является предметом следующего утверждения.

Теорема 4.3 [4; теорема 8]. Пусть $K$ - конечно порожденное поле, и пусть $n>1$ - иелое число, взаимно простое $c$ char $K$. Тогда существует набор $V$ дискретных нормирований $K$, удовлетворяющий введенным выше условиям (I) u (II), для которого ${ }_{n} \operatorname{Br}(K)_{V}$ конечна.

Наше доказательство этой теоремы, набросок которого будет дан ниже, опиралось на анализ точной последовательности для группы Брауэра кривой. Впоследствии Ж.-Л. Кольё-Телен указал нам, что утверждение о конечности можно вывести из результатов об этальных когомологиях. Более подробно, в этом доказательстве поле $K$ представляется в виде поля рациональных функций на гладкой арифметической схеме $X$, на которой $n$ обратимо. Затем используется теорема конечности Делиня для этальных когомологий конструктивных пучков (теорема 1.1 в главе "Théorèmes de finitude" в [13]), которая показывает, что в этом случае $n$-кручение этальной группы Брауэра конечно. С другой стороны, по теореме Габбера о чистоте (purity theorem; доказательство можно найти в [18], а история вопроса описана в [10; с. 153] и [9; обсуждение после теоремы 4.2]) последняя группа совпадает с неразветвленной группой Брауэра поля $K$ относительно множества $V$ дискретных нормирований $K$, ассоциированных с простыми дивизорами в $X$ (см. более подробно в [4]). Будем называть такое множество нормирований дивизориалъным. Заметим, что это рассуждение дает определенную свободу в выборе $V$ : например, $X$ может быть заменено на открытую подсхему, что позволяет удалить из $V$ любое конечное множество. Это обстоятельство дает возможность несколько упростить доказательство конечности рода. Действительно, для данной алгебры с делением $D$ мы можем выбрать такое множество $V$, что $D$ будет неразветвленной относительно всех нормирований из $V$. Тогда из леммы 4.2 нам нужен только тот факт, что любой класс $\left[D^{\prime}\right] \in \operatorname{gen}(D)$ является неразветвленным относительно всех нормирований $v \in V$ (для произвольных алгебраических групп см. теорему 6.7). Из этого сразу следует, что

$$
|\operatorname{gen}(D)| \leqslant\left|{ }_{n} \operatorname{Br}(K)_{V}\right|<\infty .
$$

Основным недостатком здесь является то, что это рассуждение не дает явных оценок порядка рода.

С другой стороны, наше первоначальное доказательство теоремы 4.3, в принципе, позволяет получить явные оценки порядка $\operatorname{gen}(D)$ в определенных случаях. Здесь мы только опишем основные идеи, отсылая за деталями к [6]. Для 
простоты предположим, что $K$ - конечно порожденное поле характеристики 0 . Тогда $K$ может быть реализовано как поле функций $k(C)$ гладкой проективной геометрически неприводимой кривой $C$ над полем $k$, которое является чисто трансцендентным расширением числового поля $P$. Пусть $V_{0}-$ множество геометрических плейсов поля $K$. Хорошо известно (см., например, [29]), что геометрическая группа Брауэра $\operatorname{Br}(K)_{\text {ur }}=\operatorname{Br}(K)_{V_{0}}$ включается в точную последовательность

$$
\operatorname{Br}(k) \stackrel{\iota k}{\longrightarrow} \operatorname{Br}(k(C)) \text { ur } \stackrel{\omega}{\longrightarrow} H^{1}(k, J) / \Phi(C, k),
$$

где $\iota_{k}$ - естественное отображение, $J$ - якобиан кривой $C$ и $\Phi(C, k)$ - некоторая конечная циклическая подгруппа в $H^{1}(k, J)$ (при этом, если $C(k) \neq \varnothing$, то $\Phi(C, k)=0$ и последовательность (11) расщепляется). В качестве требуемого множества $V$ дискретных нормирований $K$ возьмем объединение $V_{0}$ с множеством $V_{1}$ продолжений на $K$ подходящего множества нормирований поля $k$ (если $k$ - числовое поле, то $V_{1}$ состоит из продолжений на $K$ почти всех неархимедовых нормирований поля $k$ ). Тогда ${ }_{n} \operatorname{Br}(K)_{V} \subset{ }_{n} \operatorname{Br}(K)_{\text {ur }}$ и доказательство теоремы 4.3 сводится к проверке конечности $\iota_{k}^{-1}\left({ }_{n} \operatorname{Br}(K)_{V}\right)$ и $\omega\left({ }_{n} \operatorname{Br}(K)_{V}\right)$.

Доказательство конечности $\iota_{k}^{-1}\left({ }_{n} \operatorname{Br}(K)_{V}\right)$ использует свойства нашей реализации $K$ в виде $k(C)$, точную последовательность Фаддеева, связывающую группы Брауэра полей $k$ и $P$, и $(\mathrm{ABHN})$. Наше доказательство конечности $\omega\left({ }_{n} \operatorname{Br}(K)_{V}\right)$ напоминает доказательство слабой теоремы Морделла-Вейля и заключается в анализе неразветвленных классов когомологий. Обратим внимание, что некоторые модификации этого рассуждения позволяют показать, что из построенного множества $V$ можно удалить любое конечное подмножество, не нарушая конечности ${ }_{n} \operatorname{Br}(K)_{V}$.

В завершение этого раздела мы приведем в качестве иллюстрации набросок доказательства теоремы 4.3 в случае, когда $K$ - поле функций эллиптической кривой над числовым полем и $n=2$ (см. [5; 4$])$. Рассматривается следующая ситуация. Пусть $k$ - числовое поле и $E$ - эллиптическая кривая над $k$, заданная уравнением Вейерштрасса

$$
y^{2}=f(x), \quad \text { где } \quad f(x)=x^{3}+\alpha x^{2}+\beta x+\gamma .
$$

Обозначим дискриминант $f$ через $\delta \neq 0$. Будем предполагать, что 2 -кручение эллиптической кривой $E$ рационально над $k$, т. е. многочлен $f$ имеет в $k$ три (различных) корня:

$$
f(x)=(x-a)(x-b)(x-c) .
$$

Пусть

$$
K:=k(E)=k(x, y)
$$

- поле функций на $E$. Поскольку $E(k) \neq \varnothing$ и $E$ совпадает со своим якобианом, последовательность (11) приводит к следующей точной последовательности:

$$
0 \rightarrow{ }_{2} \operatorname{Br}(k) \rightarrow{ }_{2} \operatorname{Br}(K) \text { ur } \stackrel{\omega}{\longrightarrow}{ }_{2} H^{1}(k, E) \rightarrow 0 .
$$


Эта последовательность расщепляется, и сечение для $\omega$ строится следующим образом. Последовательность Куммера

$$
0 \rightarrow E[2] \rightarrow E \stackrel{\times 2}{\longrightarrow} E \rightarrow 0
$$

порождает точную последовательность когомологий

$$
0 \rightarrow E(k) / 2 E(k) \rightarrow H^{1}(k, E[2]) \stackrel{\sigma}{\rightarrow}{ }_{2} H^{1}(k, E) \rightarrow 0 .
$$

Поскольку $E[2]$ и $\mathbb{Z} / 2 \mathbb{Z} \times \mathbb{Z} / 2 \mathbb{Z}$ изоморфны как модули над группой Галуа, мы имеем

$$
H^{1}(k, E[2]) \simeq k^{\times} /\left(k^{\times}\right)^{2} \times k^{\times} /\left(k^{\times}\right)^{2} .
$$

Рассмотрим отображение

$$
\nu: H^{1}(k, E[2]) \rightarrow{ }_{2} \operatorname{Br}(k(E))_{\mathrm{ur}}, \quad(r, s) \mapsto\left[\left(\frac{r, x-b}{K}\right) \otimes_{K}\left(\frac{s, x-c}{K}\right)\right] .
$$

Можно проверить, что $\omega \circ \nu=\sigma$ и $\nu(\operatorname{ker} \sigma)=0$, откуда и получается требуемое сечение

$$
\varepsilon:{ }_{2} H^{1}(k, E) \rightarrow{ }_{2} \operatorname{Br}(k(E))_{\mathrm{ur}} .
$$

Это приводит к следующему описанию группы Брауэра, которое справедливо над любым полем $k$ характеристики $\neq 2,3$.

Теорема 4.4 [3; теорема 3.6]. Предположим, что 2-кручение эллиптической кривой $E$, заданной уравнением (12), рачионально над $k$, т.е.

$$
f(x)=(x-a)(x-b)(x-c), \quad \text { әде } a, b, c \in k .
$$

Тогда

$$
{ }_{2} \operatorname{Br}(K) \text { ur }={ }_{2} \operatorname{Br}(k) \oplus I,
$$

где ${ }_{2} \operatorname{Br}(k)$ отождествляется с подгруппой в ${ }_{2} \operatorname{Br}(K)$ с помощью канонического отображения $\operatorname{Br}(k) \rightarrow \operatorname{Br}(K)$, a $I \subset{ }_{2} \operatorname{Br}(K)$ ur - такая подгруппа, что каждый элемент из I может быть представлен бикватернионной алгеброй вида

$$
\left(\frac{r, x-b}{K}\right) \otimes_{K}\left(\frac{s, x-c}{K}\right)
$$

для некоторых $r, s \in k^{\times}$.

Как мы уже упоминали выше, нужное нам множество $V$ получается объединением множества $V_{0}$ геометрических плейсов и множества $V_{1}$, которое состоит из продолжений на $K$ почти всех неархимедовых нормирований поля $k$ и описывается следующим образом. Для $s \in k^{\times}$обозначим через $V^{k}(s)$ конечное множество $\left\{v \in V^{k} \backslash V_{\infty}^{k} \mid v(s) \neq 0\right\}$. Зафиксируем конечное множество нормирований $S \subset V^{k}$, содержащее $V_{\infty}^{k} \cup V^{k}(2) \cup V^{k}(\delta)$ и все те неархимедовы $v \in V^{k}$, для которых хотя бы одно из чисел $v(\alpha), v(\beta)$ или $v(\gamma)$ отрицательно. Для неархимедова $v \in V^{k}$ обозначим через $\widetilde{v}$ его продолжение на $F:=k(y)$, задаваемое формулой

$$
\widetilde{v}(p(y))=\min _{a_{i} \neq 0} v\left(a_{i}\right), \quad \text { где } \quad p(y)=a_{n} y^{n}+\cdots+a_{0} \in k[y], \quad p \neq 0 .
$$


Заметим, что $K$ - кубическое расширение $F$. Можно показать, что для $v \in$ $V^{k} \backslash S$ нормирование $\widetilde{v}$ имеет единственное продолжение на $K$, которое мы будем обозначать через $w=w(v)$ (см. [5; лемма 4.5]). Мы определим

$$
V_{1}=\left\{w(v) \mid v \in V^{k} \backslash S\right\}
$$

и положим $V=V_{0} \cup V_{1}$.

Предложение 4.5. Неразветвленная группа Брауэра ${ }_{2} \operatorname{Br}(K)_{V}$ конечна.

ДокАЗАТЕЛЬСтво (нАБРосок). Мы рассмотрим ветвление относительно нормирований из $V_{1}$ отдельно для константных и бикватернионных компонент элементов ${ }_{2} \operatorname{Br}(K)$ ur в смысле разложения, заданного теоремой 4.4. Представим $[D] \in{ }_{2} \operatorname{Br}(K)_{V}$ в виде

$$
[D]=\left[\Delta \otimes_{k} K\right] \otimes_{K}\left[\left(\frac{r, x-b}{K}\right) \otimes_{K}\left(\frac{s, x-c}{K}\right)\right],
$$

где $[\Delta] \in{ }_{2} \operatorname{Br}(k)$ - алгебра кватернионов и $r, s \in k^{\times}$. Используя свойства отображения коограничения, а также явное описание отображений вычета в этой ситуации (см. [5; предложение 4.7 и лемма 4.8]), можно показать, что для любого $v \in V^{k} \backslash S$ алгебра кватернионов $\Delta$ неразветвлена относительно $v$ и

$$
v(r), v(s) \equiv 0 \quad(\bmod 2) .
$$

Из этого следует конечность ${ }_{2} \operatorname{Br}(K)_{V}$. Действительно, как мы видели в примере 4.1, (b), неразветвленная группа Брауэра ${ }_{2} \operatorname{Br}(k)_{V^{k} \backslash S}$ конечна, и, следовательно, имеется только конечное число возможностей для $[\Delta]$. С другой стороны, как известно, из конечности числа классов и конечной порожденности группы $S$-единиц вытекает, что образ множества

$$
P(k, S)=\left\{x \in k^{\times} \mid v(x) \equiv 0(\bmod 2) \text { для всех } v \in V^{k} \backslash S\right\}
$$

при каноническом отображении $k^{\times} \rightarrow k^{\times} /\left(k^{\times}\right)^{2}$ конечен, откуда вытекает конечность числа возможных бикватернионных компонент.

Мы теперь дадим набросок когомологического доказательства условия (16), которое напоминает рассуждение, используемое в стандартном доказательстве слабой теоремы Морделла-Вейля (см., например [46; гл. VIII, §2]). Сначала напомним следующее определение. Пусть $v \in V^{k} \backslash V_{\infty}^{k}$. Будем говорить, что класс $x \in H^{1}(k, E[2])$ неразветвлен относительно $v$, если

$$
x \in \operatorname{ker}\left(H^{1}(k, E[2]) \stackrel{\operatorname{res}_{v}}{\longrightarrow} H^{1}\left(k_{v}^{\mathrm{ur}}, E[2]\right)\right),
$$

где $k_{v}^{\mathrm{ur}}$ - максимальное неразветвленное расширение пополнения $k_{v}$ и $\operatorname{res}_{v}-$ отображение ограничения. Далее, для множества $U \subset V^{k} \backslash V_{\infty}^{k}$ мы определяем соответствующую группу неразветвленных когомологий как

$$
H^{1}(k, E[2])_{U}=\bigcap_{v \in U} \operatorname{ker}\left(H^{1}(k, E[2]) \stackrel{\operatorname{res}_{v}}{\longrightarrow} H^{1}\left(k_{v}^{\mathrm{ur}}, E[2]\right)\right) .
$$


Показывается, что если $x \in I$ содержится в ${ }_{2} \operatorname{Br}(K)_{V}$, то выполняется включение

$$
\sigma^{-1}(\omega(x)) \subset H^{1}(k, E[2])_{V^{k} \backslash S},
$$

где $\sigma: H^{1}(k, E[2]) \rightarrow{ }_{2} H(k, E)$ - отображение из точной последовательности $(14)$. $\mathrm{C}$ другой стороны, хорошо известно, что при каноническом изоморфизме

$$
H^{1}\left(k_{v}^{\mathrm{ur}}, \mathbb{Z} / 2 \mathbb{Z}\right) \simeq\left(k_{v}^{\mathrm{ur}}\right)^{\times} /\left(k_{v}^{\mathrm{ur}}\right)^{\times^{2}}
$$

смежный класс $a\left(k_{v}^{\mathrm{ur}}\right)^{\times 2} \in\left(k_{v}^{\mathrm{ur}}\right)^{\times} /\left(k_{v}^{\mathrm{ur}}\right)^{\times 2}$ соответствует характеру

$$
\chi_{a}: \operatorname{Gal}\left(\left(k_{v}^{\mathrm{ur}}\right)^{\mathrm{sep}} / k_{v}^{\mathrm{ur}}\right) \rightarrow \mathbb{Z} / 2 \mathbb{Z}
$$

с ядром ker $\chi_{a}=\operatorname{Gal}\left(\left(k_{v}^{\mathrm{ur}}\right)^{\mathrm{sep}} / k_{v}^{\mathrm{ur}}(\sqrt{a})\right)$ (см., например, [21; предложение 4.3.6 и следствие 4.3.9]). Из этого следует, что если $a\left(k_{v}^{\mathrm{ur}}\right)^{\times 2}$ соответствует классу когомологий, неразветвленному в $v$, то $\sqrt{a} \in k_{v}^{\mathrm{ur}}$ и, следовательно, $v(a) \equiv 0$ $(\bmod 2)$ (см. [28; предложение 1.3]). Сопоставляя это замечание с приведенным выше описанием геометрической группы Брауэра, мы видим, что неразветвленность

$$
\left[\left(\frac{r, x-b}{K}\right) \otimes_{K}\left(\frac{s, x-c}{K}\right)\right] \in I
$$

относительно $w(v) \in V_{1}$ влечет (16).

Отметим, что рассуждение, набросок которого мы только что привели, не только дает конечность неразветвленной группы Брауэра ${ }_{2} \operatorname{Br}(K)_{V}$, но и позволяет оценить ее порядок. Точнее, имеется следующий результат.

ТеОрема 4.6. Для любого конечного множества нормирований $S$ поля $k$ указанного выше вида неразветвленная группа Брауэра ${ }_{2} \operatorname{Br}(K)_{V}$ является конечной группой, порядок которой делит

$$
2^{|S|-t} \cdot\left|{ }_{2} \mathrm{Cl}_{S}(k)\right|^{2} \cdot\left|U_{S}(k) / U_{S}(k)^{2}\right|^{2},
$$

где $t=c+1$ и $c$ - число комплексных классов эквивалентности нормирований $k, a \mathrm{Cl}_{S}(k)$ и $U_{S}(k)$ обозначают соответственно группу классов идеалов $u$ группу единии, кольиа $\mathscr{O}_{k}(S)$ элементов поля $k$, целых относительно всех $v \in V^{k} \backslash S$.

ПримеР 4.7. Рассмотрим эллиптическую кривую $E$ над $\mathbb{Q}$, заданную уравнением $y^{2}=x^{3}-x$. Здесь $\delta=4$, так что $S=\{\infty, 2\}$. Кроме того,

$$
|S|-t=1, \quad \mathrm{Cl}_{S}(\mathbb{Q})=1 \quad \text { и } \quad U_{S}(\mathbb{Q})=\{ \pm 1\} \times \mathbb{Z} .
$$

Таким образом, согласно теореме 4.6 и неравенству (10), для любой алгебры кватернионов с делением $D$ над $K=\mathbb{Q}(E)$ выполняется оценка $|\operatorname{gen}(D)| \leqslant$ $2 \cdot 4^{2}=32$. 


\section{5. Некоторые модификации понятия рода}

В этом разделе мы обсудим несколько естественных вариаций понятия рода алгебры с делением, которые оказываются полезными в разных ситуациях.

Во-первых, можно рассмотреть локальный род, который уже неявно появлялся в доказательстве леммы 4.2. Пусть $K$ - поле и $V$ - множество дискретных нормирований поля $K$. Определим локалъный род gen $_{V}(D)$ центральной алгебры с делением $D$ степени $n$ над полем $K$ как множество классов $\left[D^{\prime}\right] \in \operatorname{Br}(K)$, представимых такой центральной алгеброй с делением $D^{\prime}$ степени $n$ над $K$, что для любого $v \in V$ выполняется следующее: если

$$
D \otimes_{K} K_{v}=M_{\ell}(\mathscr{D}) \quad \text { и } \quad D^{\prime} \otimes_{K} K_{v}=M_{\ell^{\prime}}\left(\mathscr{D}^{\prime}\right),
$$

где $\mathscr{D}$ и $\mathscr{D}^{\prime}$ - центральные алгебры с делением над пополнением $K_{v}$, то $\ell=\ell^{\prime}$, а $\mathscr{D}$ и $\mathscr{D}^{\prime}$ имеют одинаковые максимальные сепарабельные подполя. Лемма 2.3 из [40] (см. также [20; лемма 3.1]) показывает, что для любого множества $V$ дискретных нормирований поля $K$ имеет место включение

$$
\operatorname{gen}(D) \subset \operatorname{gen}_{V}(D) .
$$

Пусть теперь $V$ - множество дискретных нормирований, удовлетворяющих условиям (I) и (II) из раздела 4 для некоторого целого числа $n>1$. Тогда рассуждения из раздела 4 показывают, что если $n$ взаимно просто $c$ char $K$ u неразветвленная группа Брауэра ${ }_{n} \operatorname{Br}(K)_{V}$ конечна, то множество gen $_{V}(D)$ конечно для любой центральной алгебры с делением $D$ степени $n$ над $K$. Действительно, как показывает доказательство леммы 4.2 , уже условие $\left[D^{\prime}\right] \in$ $\operatorname{gen}_{V}(D)$ влечет, что для любого $v \in V$ характеры $\chi_{v}=\rho_{v}([D])$ и $\chi_{v}^{\prime}=\rho_{v}\left(\left[D^{\prime}\right]\right)$ имеют одинаковое ядро. Это замечание приводит в конечном счете к оценке $(10)$, в которой $\operatorname{gen}(D)$ заменен на локальный род $\operatorname{gen}_{V}(D)$.

Далее, как мы уже упоминали в замечании 3.7, (b), Д. Крашен и К. Маккини [27] изучали алгебры с делением, имеющие одни и те же конечномерные поля разложения. С этой целью для конечномерной центральной алгебры с делением $D$ над полем $K$ определим gen' $(D)$ как набор классов $\left[D^{\prime}\right] \in \operatorname{Br}(K)$, удовлетворяющих следующему условию: конечное расширение поля $L / K$ расщепляет $D$ тогда и только тогда, когда оно расщепляет $D^{\prime}$ (обозначения в [27] немного отличаются). Понятно, что

$$
\operatorname{gen}^{\prime}(D) \subset \operatorname{gen}(D) .
$$

Основным результатом работы [27] является следующее утверждение. Если $p-$ простое число, отличное от $\operatorname{char} K$, и

$$
\left|\operatorname{gen}^{\prime}(\Delta)\right|<\infty
$$

для всех $[\Delta] \in{ }_{p} \operatorname{Br}(K)$, то

$$
\left|\operatorname{gen}^{\prime}(D)\right|<\infty
$$

для всех $[D] \in{ }_{p} \operatorname{Br}(K(t))$ (аналогичный результат для $\operatorname{gen}(D)$ был получен в [5], но формально эти результаты не следуют один из другого). 
Третье понятие, которое нам хотелось бы кратко обсудить, это так называемый односторонний (или асимметричный) род, который был введен в [26]. Следуя [26], для двух центральных алгебр с делением $D$ и $D^{\prime}$ одинаковой степени над полем $K$ будем писать

$$
D \leqslant D^{\prime}
$$

если любое максимальное подполе $P / K$ алгебры $D$ вкладывается над $K$ в алгебру $D^{\prime}$. Для алгебры с делением $D$ степени $n$ над $K$ определим односторонний род gen $^{1}(D)$ как набор классов $\left[D^{\prime}\right] \in \operatorname{Br}(K)$, где $D^{\prime}$ - такая центральная алгебра с делением степени $n$, что $D \leqslant D^{\prime}$. Мы отсылаем читателя к [26] за более подробным обсуждением этого понятия, а здесь только прокомментируем различия в свойствах ветвлений, возникающие в ходе анализа двустороннего и одностороннего рода.

Согласно лемме 4.2 , если $\left[D^{\prime}\right] \in \operatorname{gen}(D)$, то дискретное нормирование $v$ поля $K$ ветвится в $D$ тогда и только тогда, когда оно ветвится в $D^{\prime}$ (при условии, что отображение вычета $\rho_{v}$ определено). Для одностороннего рода ситуация более сложная. Для числового поля $K$ из замечаний, предшествующих доказательству предложения 3.1, вытекает, что отношение $D \leqslant D^{\prime}$ влечет включение $R\left(D^{\prime}\right) \subset R(D)$ для соответствующих множеств разветвленных нормирований. $\mathrm{C}$ другой стороны, пусть $K$ - поле формальных степенных рядов $\mathbb{R}((x))$ со стандартным дискретным нормированием $v$. Рассмотрим следующие два тела кватернионов над $K$ :

$$
D_{1}=\left(\frac{-1,-1}{K}\right) \quad \text { и } \quad D_{2}=\left(\frac{-1, x}{K}\right) .
$$

Тогда тело $D_{1}$ неразветвлено относительно $v$ и его алгебра вычетов изоморфна телу обычных гамильтоновых кватернионов $\mathbb{H}$ над $\mathbb{R}$, в то время как тело $D_{2}$ ветвится. Каждое квадратичное подполе $L$ тела $D_{1}$ неразветвлено над $K$, и, поскольку $\mathbb{C}$ является единственным нетривиальным конечным расширением поля вычетов $\bar{K}_{v}=\mathbb{R}$, можно сделать вывод, что $L$ изоморфно $K(i)=\mathbb{C}((x))$ (где $\left.i^{2}=-1\right)$. Поскольку $\mathbb{C}((x))$ содержится в $D_{2}$, мы видим, что на самом деле любое максимальное подполе $D_{1}$ можно вложить в $D_{2}$. Это означает, что $D_{1} \leqslant D_{2}$ и, следовательно, $\left[D_{2}\right] \in \operatorname{gen}^{1}\left(D_{1}\right)$. Тем не менее $v$ ветвится в $D_{2}$, но не в $D_{1}$.

Результаты работы [26] показывают, что эта конструкция доставляет в некотором смысле универсальный пример, который приводит к таким аномалиям, связанным с ветвлением. Чтобы сформулировать точные утверждения, нам потребуется ввести некоторые термины и обозначения. В [26] конечномерная центральная алгебра с делением $D$ над полем $K$ называлась варъируемой (varied), если не существует нетривиального циклического расширения $P / K$, которое вкладывалось бы в каждое максимальное подполе алгебры $D$ (заметим, что это свойство достаточно проверить для циклических расширений $P / K$ простой степени). Например, известно, что если поле $K$ конечно порождено над глобальным полем, то любая центральная алгебра с делением $D$ над $K$ варьируема (см. [26; теорема 1]). Для центральной алгебры с делением $D$ над полем $K$, 
полным относительно дискретного нормирования, обозначим через $\bar{D}$ алгебру вычетов и через $\bar{E}_{D}$ ее центр; пусть $E_{D}$ - единственное (с точностью до изоморфизма) неразветвленное подполе $D$ с полем вычетов $\bar{E}_{D}$ (в $[26]$ последнее называется полем ветвления D).

ПреДлОжЕНИЕ 5.1 [26; предложение 4]. Пусть $K$ - поле, полное относительно дискретного нормирования $v$, и пусть $D$ и $D^{\prime}$ - централъные алгебры с делением над $K$ одинаковой степени такие, что $D \leqslant D^{\prime}$. Если алгебра $\bar{D} / \bar{E}_{D}$ варъируема над $\bar{E}_{D}$, mо $E_{D}=E_{D^{\prime}}$.

Для того чтобы извлечь из этого результата информацию о ветвлении, предположим, что существует отображение вычета

$$
\rho_{v}:{ }_{n} \operatorname{Br}(K) \rightarrow \operatorname{Hom}\left(\mathscr{G}^{(v)}, \mathbb{Z} / n \mathbb{Z}\right),
$$

где $n=\operatorname{deg} D$. Как уже отмечалось, хорошо известно, что для $\chi_{v}=\rho_{v}([D])$ поле $\bar{E}_{D}$ совпадает с подполем сепарабельного замыкания поля вычетов $\bar{K}_{v}$, отвечающим подгруппе ker $\chi_{v}$. Таким образом, согласно предложению 5.1 , утверждение леммы 4.2 справедливо, как только $D \leqslant D^{\prime}$, при условии, что алгебра $\bar{D} / \bar{E}_{D}$ варьируема; в частности, $D$ и $D^{\prime}$ одновременно ветвятся или не ветвятся относительно $v$. Приведенный выше пример алгебр кватернионов над $K=\mathbb{R}((x))$ показывает, что предположение о варьируемости нельзя отбросить - заметим, что $E_{D_{1}}=\mathbb{R}((x))$, в то время как $E_{D_{2}}=\mathbb{C}((x))$. С другой стороны, оказывается, что все ситуации, в которых алгебра с делением не является варьируемой, вплотную связаны с этим примером.

ПредлОжениЕ 5.2 [26; §3]. (а) Пусть D - конечномерная чентральная алгебра с делением над полем $K$, не являющаяся варьируемой. Тогда $K$ - пифагорово поле и

$$
D=\left(\frac{-1,-1}{K}\right) \otimes_{K} D^{\prime}
$$

для некоторой конечномерной централъной алгебры с делением $D^{\prime}$ над $K$.

(b) Пусть $K$ - поле, полное относительно дискретного нормирования, и пусть $D, D^{\prime}$ - такие центральные алгебры с делением над $K$ одинаковой степени, что $D \leqslant D^{\prime}$. Тогда $E_{D} E_{D^{\prime}} \subseteq E_{D}(\sqrt{-1})$. Если $E_{D} \subsetneq E_{D^{\prime}}=E_{D}(\sqrt{-1})$, mо степень $\left[E_{D}: K\right]$ нечетна и $K=F((x))$ для некоторого пифагорова поля $F$.

Напомним, что поле $F$ называется пифагоровым, если сумма двух квадратов в $F$ снова является квадратом.

\section{6. Род алгебраической группы}

Мы завершим эту статью кратким обзором проводимых исследований, цель которых - распространить методы и результаты, разработанные в контексте алгебр с делением (см. разделы 3-5), на абсолютно почти простые алгебраические группы всех типов. В этой ситуации понятие алгебр с делением, обладающих одинаковыми максимальными подполями, заменяется понятием алгебраических групп с одинаковыми максимальными торами. Более точно, пусть $G_{1}$ и 
$G_{2}$ - абсолютно почти простые группы над полем $K$. Будем говорить, что $G_{1}$ и $G_{2}$ имеют одни и те же классы $K$-изоморфизма (соответственно $K$-изогении) максимальных $K$-торов, если каждый максимальный $K$-тор $T_{1}$ группы $G_{1}$ $K$-изоморфен (соответственно $K$-изогенен) некоторому максимальному $K$-тору $T_{2}$ группы $G_{2}$, и наоборот. Далее, пусть $G$ - алгебраическая $K$-группа и $K^{\mathrm{sep}}$ сепарабельное замыкание поля $K$. Напомним, что алгебраическая $K$-группа $G^{\prime}$ называется $K$-формой (или, более точно, $K^{\mathrm{sep}} / K$-формой) группы $G$, если $G$ и $G^{\prime}$ изоморфны над $K^{\mathrm{sep}}$ (см., например, [44; гл. III, §1] или [35; гл. II, §2]). Например, для любой центральной алгебры с делением $D$ степени $n$ над $K$ существует $K^{\mathrm{sep}}$-изоморфизм $D \otimes_{K} K^{\mathrm{sep}} \simeq M_{n}\left(K^{\mathrm{sep}}\right)$, что означает, что алгебраическая $K$-группа $G=\mathrm{SL}_{1, D}$, ассоциированная с группой элементов из $D$, имеющих приведенную норму 1 , является $K$-формой $\mathrm{SL}_{n}$.

ОПРЕДЕЛЕНИЕ 6.1. Пусть $G$ - абсолютно почти простая алгебраическая группа над полем $K$. Множество классов $K$-изоморфизма $K$-форм $G^{\prime}$ группы $G$, имеющих те же классы изоморфизма максимальных $K$-торов, что и $G$, называется $\left(K\right.$-)родом группы $G$ (обозначение: $\operatorname{gen}_{K}(G)$ или просто $\operatorname{gen}(G)$, если это не может привести к недоразумению).

Нужно отметить, что для конечномерной центральной $K$-алгебры с делением $D$ только максимальные сепарабелъные подполя в $D$ приводят к максимальным $K$-торам соответствующей группы $G=\mathrm{SL}_{1, D}$. Таким образом, мы видим задним числом, что для согласования определений $\operatorname{gen}(D)$ и $\operatorname{gen}(G)$ первое из них, вероятно, следовало бы переформулировать в терминах максимальных сепарабельных подполей. Это изменение не повлияло бы на результаты разделов 3 и 4, ибо там рассматривались алгебры, степень которых взаимно проста c char $K$, но пока непонятно, оказало бы это влияние на общий случай. В данной статье нас будут в основном интересовать простые алгебраические группы с одинаковыми классами изоморфизма максимальных торов, хотя анализ слабой соизмеримости плотных по Зарискому подгрупп, который связан с геометрическими приложениями (см. [36]), требует иногда рассмотрения простых групп с одинаковыми классами изогении максимальных торов.

Как и в случае алгебр с делением, мы рассмотрим следующие два вопроса.

\section{Вопрос $1^{\prime}$. Когда множество gen $_{K}(G)$ сводится к одному элементу?}

Это означает, что среди $K$-групп данного типа группа $G$ однозначно определяется с точностью до $K$-изоморфизма своими максимальными $K$-торами.

Вопрос $2^{\prime}$. Когда множество $\operatorname{gen}_{K}(G)$ конечно?

На данный момент полностью рассмотрен только случай абсолютно почти простых алгебраических групп над числовыми полями.

Tеорема 6.2 (ср. [36; теорема 7.5]). (а) Пусть $G_{1} u G_{2}$ - связные абсолютно почти простые алгебраические группы, определенные над числовым полем $K$, и пусть $L_{i}$ - минимальное расширение Галуа поля $K$, над которым $G_{i}$ становится внутренней формой расщепимой группь. Если $G_{1} u G_{2}$ имеют 
одинаковые классы $K$-изогении максимальных $K$-торов, то либо $G_{1}$ и $G_{2}$ имеют один и тот же тип, либо одна из групп имеет тип $\mathrm{B}_{n}$, а другая - тип $\mathrm{C}_{n}$ $(n \geqslant 3), u$, кроме того, $L_{1}=L_{2}$.

(b) Зафиксируем абсолютно почти простую $K$-группу. Тогда множество классов изоморфизма всех абсолютно почти простьх $K$-групп $G^{\prime}$ с теми же классами $K$-изогении максимальных $K$-торов, что и $G$, конечно.

(c) Зафиксируем абсолютно почти простую односвязную $K$-группу $G$, тип которой отличен от $\mathrm{A}_{n}, \mathrm{D}_{2 n+1}(n>1) u \mathrm{E}_{6}$. Тогда любая K-форма $G^{\prime}$ группъ $G$ (другими словами, любая абсолютно почти простая односвязная $K$-группа $G^{\prime}$ того же типа, что и $\left.G\right)$, которая имеет те же классы $K$-изогении максимальных $K$-торов, что и $G$, является $K$-изоморфной $G$.

Для типов, исключенных в формулировке части (с) теоремы, ее утверждение действительно может нарушаться. А именно, конструкция, описанная в [36; $\S 9]$, позволяет построить для каждого из этих типов неизоморфные абсолютно почти простые односвязные $K$-группы $G_{1}$ и $G_{2}$ данного типа, которые имеют одинаковые классы $K$-изоморфизма максимальных $K$-торов. Группы типов $\mathrm{B}_{n}$ и $\mathrm{C}_{n}$, имеющие одни и те же классы $K$-изоморфизма максимальных $K$-торов, проанализированы в [19].

Укажем теперь на дополнительные трудности, которые возникают при изучении $\operatorname{gen}(G)$ даже в случае групп вида $G=\mathrm{SL}_{m, D}$, где $D$ - центральная алгебра с делением степени $n$. Напомним, что $G$ является односвязной внутренней формой типа $\mathrm{A}_{\ell}$, где $\ell=m n-1$, и что все внутренние формы этого типа получаются таким образом (см. [35; предложение 2.17]). Каждый максимальный $K$-тор такой группы $G$ является норменным тором

$$
\mathrm{R}_{E / K}^{(1)}\left(G_{m}\right)=\mathrm{R}_{E / K}\left(G_{m}\right) \cap G,
$$

отвечающим некоторой этальной подалгебре $E$ матричной алгебры $M_{m}(D)$, где $G_{m}$ - одномерный расщепимый тор, a $\mathrm{R}_{E / K}$ - функтор ограничения основного поля. Проблема заключается в том, что наличие $K$-изоморфизма между норменными торами $\mathrm{R}_{E_{1} / K}^{(1)}\left(G_{m}\right)$ и $\mathrm{R}_{E_{2} / K}^{(1)}\left(G_{m}\right)$, вообще говоря, не влечет изоморфности $E_{1}$ и $E_{2}$, даже если эти алгебры являются полями. По этой причине довольно сложно установить взаимоотношение между $\operatorname{gen}(G)$ и $\operatorname{gen}(D)$, которое позволило бы использовать результаты разделов 3-4. Тем не менее, используя дополнительные соображения, можно доказать следующую теорему, которая аналогична теоремам 3.3 и 3.6 для алгебр с делением.

Теорема 6.3 (см. [5; теорема 5.3]). (а) Пусть D - центральная алгебра с делением экспоненты 2 над $K=k\left(x_{1}, \ldots, x_{r}\right)$, где $k$ - либо числовое поле, либо конечное поле характеристики $\neq 2$. Тогда для любого $m \geqslant 1$ род группь $G=\mathrm{SL}_{m, D}$ сводится $\kappa$ одному элементу.

(b) Пусть $G$ - абсолютно почти простая односвязная алгебраическая группа внутреннего типа $\mathrm{A}_{\ell}$ над конечно порожденным полем $K$, характеристика которого либо равна нулю, либо не делит $\ell+1$. Тогда множество $\operatorname{gen}(G)$ конечно. 
Доказательство теоремы 6.3 использует так называемые торь общего положения. Поскольку эти торы играют все более важную роль в различных контекстах, мы напомним связанные с ними определения и результаты.

Пусть $G$ - полупростая алгебраическая группа над полем $K$. Зафиксируем максимальный $K$-тор $T$ группы $G$ и обозначим через $\Phi=\Phi(G, T)$ соответствующую систему корней. Далее, пусть $K_{T}$ - минимальное поле разложения тора $T$ и $\Theta_{T}=\operatorname{Gal}\left(K_{T} / K\right)$ - его группа Галуа. Тогда естественное действие группы $\Theta_{T}$ на группе характеров $X(T)$ задает инъективный гомоморфизм групп

$$
\theta_{T}: \Theta_{T} \rightarrow \operatorname{Aut}(\Phi)
$$

Будем говорить, что тор $T$ является тором общего положения над $K$, если образ гомоморфизма $\theta_{T}$ содержит группу Вейля $W(\Phi)=W(G, T)$. Например, для $G=\mathrm{SL}_{m, D}$ максимальный $K$-тор $T=\mathrm{R}_{E / K}^{(1)}\left(G_{m}\right)$ является тором общего положения тогда и только тогда, когда $E$ - сепарабельное расширение поля $K$ степени $m n$ и группа Галуа его нормального замыкания совпадает с симметрической группой $S_{m n}$.

Как показывает следующий результат, в случае конечно порожденного поля $K$ всегда найдется $K$-тор общего положения с заданными локальными свойствами.

ПРЕДЛОЖЕНИЕ 6.4 (см. [37; следствие 3.2]). Пусть $G$ - абсолютно почти простая группа над конечно порожденным полем $K$. Если $v$ - дискретное нормирование $K$ и $T_{v}$-максимальныи $K_{v}$-тор группы $G$, то существует максимальный $K$-тор $T$ группы $G$, который является тором общего положения над $K$ и сопряжен тору $T_{v}$ при помощи элемента из $G\left(K_{v}\right)$.

Второй результат, который мы хотели бы упомянуть, - это своеобразная жесткость изоморфизмов между торами общего положения. Более точно, пусть $G_{1}$ и $G_{2}$ - две односвязные внутренние $K$-формы типа $\mathrm{A}_{\ell}$ и $T_{i}$ - максимальный $K$-тор общего положения в $G_{i}$ для $i=1,2$. Тогда любой $K$-изоморфизм $\varphi: T_{1} \rightarrow T_{2}$ продолжается до $K^{\mathrm{sep}}$-изоморфизма $\widetilde{\varphi}: G_{1} \rightarrow G_{2}$. (Это утверждение является следствием так называемой теоремы об изогениях, см. [36; теорема 4.2 и замечание 4.4] и [38; теорема 9.8].) Из этого вытекает, что для норменных торов общего положения $T_{i}=R_{E_{i} / K}^{(1)}\left(G_{m}\right)$ в вышеприведенных обозначениях существование $K$-изоморфизма торов $T_{1} \rightarrow T_{2}$ влечет наличие изоморфизма $K$-алгебр $E_{1} \rightarrow E_{2}$. После этих замечаний мы в состоянии дать

НАБРОСОК ДОКАЗАТЕЛЬСТВА ТЕОРЕМЫ 6.3. ПреДПоЛожим, что $K$ - конечно порожденное поле и $G^{\prime} \in \operatorname{gen}_{K}(G)$. Сначала показывается, что $G^{\prime}$ является внутренней формой над $K$ и в действительности $G^{\prime}=\mathrm{SL}_{m, D^{\prime}}$ для некоторой центральной алгебры с делением $D^{\prime}$ степени $n$ над $K$. С использованием свойства торов общего положения, доказывается, что для любого дискретного нормирования $v$ поля $K$ алгебры $D \otimes_{K} K_{v}$ и $D^{\prime} \otimes_{K} K_{v}$ имеют одни и те же классы изоморфизма максимальных этальных подалгебр. Это означает, что для любого множества $V$ дискретных нормирований поля $K$ класс $\left[D^{\prime}\right]$ лежит в локальном роде $\operatorname{gen}_{V}(D)$. С другой стороны, согласно теореме 4.3 , для конечно 
порожденного поля $K$ характеристики, взаимно простой с $n$, существует множество $V$ дискретных нормирований поля $K$, удовлетворяющее условиям (I) и (II) и такое, что неразветвленная группа Брауэра ${ }_{n} \operatorname{Br}(K)_{V}$ конечна. Как указывалось в разделе 5 , это дает конечность локального рода $\operatorname{gen}_{V}(D)$, что и завершает доказательство части (b) теоремы 6.3. Для доказательства части (a) мы запишем $K=\ell\left(x_{1}\right)$, где $\ell=k\left(x_{2}, \ldots, x_{r}\right)$, и пусть $V$ - множество дискретных нормирований поля $K$, тривиальных на $\ell$. Тогда, как и в доказательстве теоремы 3.3 , из того, что $\left[D^{\prime}\right] \in \operatorname{gen}_{V}(D)$, и точной последовательности Фаддеева вытекает, что

$$
[D]=\left[D^{\prime}\right]\left[\Delta \otimes_{\ell} K\right] \quad \text { в } \operatorname{Br}(K)
$$

для некоторой центральной алгебры с делением $\Delta$ над $\ell$. Наконец, для доказательства тривиальности $\Delta$ выберем точку $v_{0} \in V$ степени один так, чтобы обе алгебры $D$ и $D^{\prime}$ были неразветвлены относительно $v_{0}$. Пусть

$$
D \otimes_{K} K_{v_{0}}=M_{s}(\mathscr{D}) \quad \text { и } \quad D^{\prime} \otimes_{K} K_{v_{0}}=M_{s^{\prime}}\left(\mathscr{D}^{\prime}\right),
$$

где $\mathscr{D}, \mathscr{D}^{\prime}$ - центральные $K_{v_{0}}$-алгебры с делением. Тогда $s=s^{\prime}$, а $\mathscr{D}$ и $\mathscr{D}^{\prime}$ имеют одинаковые максимальные (сепарабельные) подполя. Из этого следует, что алгебры вычетов $\overline{\mathscr{D}}$ и $\overline{\mathscr{D}^{\prime}}$ являются центральными $\ell$-алгебрами с делением, имеющими одинаковые максимальные подполя. Поскольку алгебра $\overline{\mathscr{D}}$ имеет экспоненту 2 , ее род тривиален по теореме 3.3 . Из этого следует, что $[\overline{\mathscr{D}}]=\left[\overline{\mathscr{D}^{\prime}}\right]$, так что класс $[\Delta]$ тривиален. Таким образом, $D \simeq D^{\prime}$, и, следовательно, $G \simeq G^{\prime}$.

ЗАмЕчАниЕ 6.5. Из приведенного выше рассуждения видно, что над конечно порожденным полем $K$ имеет смысл рассматривать альтернативное понятие рода $\operatorname{gen}_{K}(G)$, определяемое в терминах максимальных $K$-торов общего положения.

Основываясь на последнем результате для рода внутренних форм типа $\mathrm{A}_{\ell}$ над конечно порожденными полями (теорема $6.3,(\mathrm{~b}))$, естественно предложить следующую гипотезу.

ГИПотезА 6.6. Пусть $G$ - абсолютно почти простая односвязная алгебраическая группа над конечно порожденным полем $K$, характеристика которого либо равна нулю, либо является хорошей ${ }^{3}$ для типа, к которому относится группа $G$. Тогда род $\operatorname{gen}_{K}(G)$ конечен.

Доказательство теоремы 6.3 показывает, что для анализа этой гипотезы нужно распространить методы, основанные на изучении ветвления и рассмотрении неразветвленных алгебр с делением, на абсолютно почти простые группы всех типов. Адекватной заменой понятию неразветвленной центральной алгебры с делением является понятие группы с хорошей редукиией. Предположим, что $G$ - абсолютно почти простая алгебраическая группа над полем $K$. Будем

\footnotetext{
3 Для данного типа плохими будем считать следующие характеристики: для типа $\mathrm{A}_{\ell}-$ все простые делители $(\ell+1)$, а также $p=2$ для внешних форм; для типов $\mathrm{B}_{\ell}, \mathrm{C}_{\ell}, \mathrm{D}_{\ell}-p=2$, a также $p=3$ для ${ }^{3,6} \mathrm{D}_{4}$; для типа $\mathrm{E}_{6}-p=2,3,5$; для типов $\mathrm{E}_{7}, \mathrm{E}_{8}-p=2,3,5,7$; для типов $\mathrm{F}_{4}$, $\mathrm{G}_{2}-p=2,3$. Все остальные характеристики для данного типа будем считать хорошими.
} 
говорить, что $G$ обладает хорошей редукцией относительно дискретного нормирования $v$ поля $K$, если существует редуктивная групповая схема ${ }^{4} \mathscr{G}$ над кольцом нормирования $\mathscr{O}_{v} \subset K_{v}$, общий слой $\mathscr{G} \otimes_{\mathscr{O}_{v}} K_{v}$ которой изоморфен $G \otimes_{K} K_{v}$. В этом случае мы будем обозначать через $\underline{G}^{(v)}$ редукцию $\mathscr{G} \otimes_{\mathscr{O}_{v}} \bar{K}_{v}$. Следующий результат дает обобщение леммы 4.2 для простых алгебраических групп всех типов.

ТЕОРема 6.7 [7]. Пусть $G$-абсолютно почти простая односвязная группа над полем $K$, и пусть $v$ - дискретное нормирование поля $K$. Предположим, что поле вычетов $\bar{K}_{v}$ конечно порождено и $G$ обладает хорошей редукцией относительно $v$. Тогда любая группа $G^{\prime} \in \operatorname{gen}_{K}(G)$ такюе обладает хорошей редукцией относительно $v$. При этом редукиия ${\underline{G^{\prime}}}^{(v)}$ принадлежит роду $\operatorname{gen}_{\overline{K_{v}}}\left(\underline{G}^{(v)}\right)$.

Отметим, что доказательство этого результата снова использует торы общего положения.

Предположим теперь, что на поле $K$ задано множество дискретных нормирований $V$, удовлетворяющее условию (I) (см. раздел 4), а также следующему условию:

(III) для любого нормирования $v \in V$ поле вычетов $\bar{K}_{v}$ конечно порождено.

СлЕДСТВИЕ 6.8. Если $K$ и $V$ удовлетворяют условиям (I) и (III), то для любой абсолютно почти простой односвязной алгебраической $K$-группы $G$ существует такое конечное подмножество $V_{0} \subset V$ (зависящее от $G$ ), что каждая группа $G^{\prime} \in \operatorname{gen}_{K}(G)$ обладает хорошей редукиией относительно всех $v \in V \backslash V_{0}$.

Из следствия 6.8 вытекает, что для того чтобы доказать гипотезу 6.6, достаточно показать, что каждое конечно порожденное поле $K$ обладает множеством $V$ дискретных нормирований, которое удовлетворяет условиям (I) и (III) и имеет также следующее свойство.

$(\Phi)$ Для любой абсолютно почти простой алгебраической $K$-группы $G$ такой, что $\operatorname{char} K$ является хорошей для $G$, и любого конечного подмножества $V_{0} \subset V$ множество классов $K$-изоморфизма (внутренних) $K$-форм $G^{\prime}$ группы $G$, имеющих хорошую редукцию во всех $v \in V \backslash V_{0}$, конечно.

(В этой формулировке, очевидно, можно считать $G$ квазиразложимой над $K$.) Можно ожидать, что возникшие при обсуждении теоремы 4.3 дивизориалъные множества нормирований, состоящие из дискретных нормирований поля $K$, отвечающих простым дивизорам некоторой гладкой арифметической схемы $X$ с полем рациональных функций $K$, будут обладать требуемым свойством для произвольных групп.

ГИПотеЗА 6.9. Любое дивизориальное множество $V$ дискретных нормирований конечно порожденного поля $K$ обладает свойством $(\Phi)$.

\footnotetext{
${ }^{4}$ Пусть $R$ - коммутативное кольцо и $S=\operatorname{Spec} R$. Напомним, что редуктивная групповая схема над $R$ - это такая гладкая аффинная групповая схема $G \rightarrow S$, что геометрические слои $G_{\bar{s}}-$ связные редуктивные алгебраические группы (см. [14; лекция ХІХ, определение 2.7] или [11; определение 3.1.1]).
} 
Над числовым полем $K$ утверждение гипотезы 6.9 является простым следствием теоремы конечности для когомологий Галуа (см. [44; гл. III, § 4.6]), поскольку полупростая группа над конечным расширением поля $\mathbb{Q}_{p}$, обладающая хорошей редукцией, обязательно квазиразложима (см. [35; теорема 6.7]). (Интересно, что имеются неразложимые группы над $\mathbb{Q}$, которые обладают хорошей редукцией относительно всех простых (см. [22], [12]), но не существует абелевых многообразий над $\mathbb{Q}$, которые всюду обладают гладкой редукцией [16].)

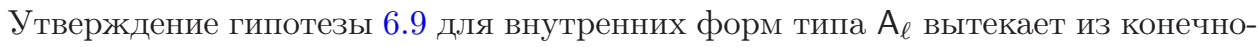
сти ${ }_{n} \operatorname{Br}(K)_{V}$. Мы также имеем следующий условный результат для спинорных групп.

Пусть $\mu_{2}=\{ \pm 1\}$. Тогда для любого дискретного нормирования $v$ поля $K$ такого, что $\operatorname{char} \bar{K}_{v} \neq 2$, и для любого $i \geqslant 1$ можно определить отображение вычета в когомологиях Галуа

$$
\rho_{v}^{i}: H^{i}\left(K, \mu_{2}\right) \rightarrow H^{i-1}\left(\bar{K}_{v}, \mu_{2}\right),
$$

продолжающее на все размерности отображение (6), введенное в разделе 4 (см., например, $[8 ; \S 3.3]$ или $[21 ; \S 6.8])$. Тогда для любого множества $V$ дискретных нормирований поля $K$ неразветвленная часть $H^{i}\left(K, \mu_{2}\right)_{V}$ определяется как $\bigcap_{v \in V} \operatorname{ker} \rho_{v}^{i}$ (разумеется, $\left.H^{2}\left(K, \mu_{2}\right)_{V}={ }_{2} \operatorname{Br}(K)_{V}\right)$.

Теорема 6.10 [7]. Пусть $K$ u V удовлетворяют гипотезе 6.9. Предположим, что для любого конечного множества $V_{0} \subset V$ неразветвленные группь когомологий $H^{i}\left(K, \mu_{2}\right)_{V \backslash V_{0}}$ конечны для всех $i \geqslant 1$. Тогда для любого $n \geqslant 5$ $u$ любого конечного подмножества $V_{0} \subset V$ множество классов $K$-изоморфизма спинорных групп $\operatorname{Spin}_{n}(q)$, где $q$ - невырожденная $n$-мерная квадратичная форма, имеющих хорошую редукиию относительно всех $v \in V \backslash V_{0}$, конечно.

Важно отметить, что спектр возможных применений гипотезы 6.9 не ограничивается конечностью рода (гипотеза 6.6) и включает, например, гипотезу конечности для слабо соизмеримых плотных по Зарискому подгрупп (см. [39; гипотеза 6.1]), а также конечность множества Тейта-Шафаревича в некоторых ситуациях. Более точно, пусть $G$ - абсолютно почти простая односвязная алгебраическая группа над полем $K$ хорошей характеристики, и пусть $V$ дивизориальное множество дискретных нормирований поля $K$. Обозначим

$$
\amalg(\bar{G}):=\operatorname{ker}\left(H^{1}(K, \bar{G}) \rightarrow \prod_{v \in V} H^{1}\left(K_{v}, \bar{G}\right)\right)
$$

множество Тейта-Шафаревича для соответствующей присоединенной группы $\bar{G}$. Выберем такое конечное подмножество $V_{0} \subset V$, что $G$ имеет хорошую редукцию во всех $v \in V \backslash V_{0}$. Пусть $\xi \in \amalg(\bar{G})$ и $G^{\prime}={ }_{\xi} G$ - соответствующая скрученная группа. По построению $G^{\prime} \simeq G$ над $K_{v}$ для всех $v \in V$, и, следовательно, $G^{\prime}$ обладает хорошей редукцией во всех $v \in V \backslash V_{0}$. Тогда, предполагая, что гипотеза 6.9 верна, мы можем сделать вывод, что для $\xi \in \amalg(\bar{G})$ группы $\xi G$ образуют конечное число классов $K$-изоморфизма; другими словами, образ $\amalg(\bar{G})$ при каноническом отображении

$$
H^{1}(K, \bar{G}) \stackrel{\lambda}{\rightarrow} H^{1}(K, \text { Aut } G)
$$


конечен. Но, поскольку $\bar{G} \simeq \operatorname{Int} G$ имеет конечный индекс в Aut $G$, отображение $\lambda$ имеет конечные слои, отсюда следует конечность $\amalg(\bar{G})$.

Заметим, что теорема 6.7 может применяться не только для исследования конечности рода, но и для доказательства того, что в некоторых ситуациях род тривиален. Например, имеет место следующее утверждение.

Теорема 6.11 [7]. Пусть $G$ - простая группа типа $\mathrm{G}_{2}$ над полем рациональных функиий $K=k(x)$, где $k$ - глобальное поле характеристики $\neq 2$. Тогда gen $_{K}(G)$ состоит из одного элемента.

Упомянем также следующий результат о стабильности.

ТЕОРема 6.12 [7]. Пусть G- абсолютно почти простая односвязная алгебрачческая группа над конечно порожденным полем $k$ характеристики нуль. Тогда для поля $k(x)$ рациональных функиий каждая группа $G^{\prime}$ из рода $\operatorname{gen}_{k(x)}\left(G \otimes_{k} k(x)\right)$ имеет вид $H \otimes_{k} k(x)$ для некоторой группы $H \in \operatorname{gen}_{k}(G)$.

Объединяя эту теорему с теоремой 6.2 , можно сделать вывод, что если $G-$ абсолютно почти простая односвязная алгебраическая группа типа, отличного от $\mathrm{A}_{\ell}(\ell>1), \mathrm{D}_{2 \ell+1}(\ell>1)$ и $\mathrm{E}_{6}$, над числовым полем $k$, то $\operatorname{gen}_{k(x)}\left(G \otimes_{k} k(x)\right)$ состоит из единственного элемента.

Мы начали эту статью с упоминания результата Ш. Амицура [2] о конечномерных центральных алгебрах с делением, имеющих одинаковые поля разложения, и с обсуждения того, как изменяется ситуация, если рассматривать только конечномерные поля разложений или только максимальные подполя. Это в конечном итоге привело к нашему определению рода алгебры с делением и, позже, рода простой алгебраической группы. Мы хотели бы завершить статью другим понятием рода, которое также основано на рассмотрении максимальных этальных подалгебр или максимальных торов, но в то же время отражает наличие бесконечномерных полей разложений, что является ключевым обстоятельством в теореме Амицура. Это (более функториальное) понятие было предложено А. С. Меркурьевым. Он определил мотивный род $\operatorname{gen}_{m}(G)$ абсолютно почти простой алгебраической $K$-группы $G$ как множество классов $K$-изоморфизма $K$-форм $G^{\prime}$ группы $G$, которые имеют одинаковые классы изоморфизма максимальных торов не только над $K$, но и над любым расширением $F$ поля $K$. Тогда из теоремы Амицура следует, что для $G=\mathrm{SL}_{1, D}$ мотивный род всегда конечен и сводится к одному элементу, если $D$ имеет экспоненту 2. Далее, согласно результату О. Т. Ижболдина [23], для невырожденных квадратичных форм $q$ и $q^{\prime}$ нечетной размерности $n$ над полем $K$ характеристики $\neq 2$ из условия

(*) q и q имеют одинаковый индекс Витта над любым расширением $F / K$ вытекает, что $q$ и $q^{\prime}$ пропорциональны (этот результат неверен для форм четной размерности). Из этого следует, что для $G=\operatorname{Spin}_{n}(q)$, где $n$ нечетно, $\left|\operatorname{gen}_{m}(G)\right|=1$. Заметим, что условие $(*)$ эквивалентно тому факту, что мотивы $q$ и $q^{\prime}$ в категории мотивов Чжоу изоморфны (см. [49], а также [50; теорема 4.18] и [25]), что и мотивировало выбор терминологии для этого понятия рода. Остальные группы пока не исследованы. 
Первый автор получил поддержку от программы Canada Research Chair и исследовательского гранта NSERC. Второй автор получил поддержку от NSF (грант DMS-1301800), BSF (грант 201049) и фонда Александра фон Гумбольдта. Третий автор получил поддержку от NSF Postdoctoral Fellowship. Часть этой работы была написана во время конференции Second Joint International Meeting of the AMS and the IMU в Израиле (июнь 2014). Авторы благодарят университеты Бар-Илана и Тель-Авива за теплый прием.

\section{Список литературы}

[1] S. A. Amitsur, "Generic splitting fields of central simple algebras", Ann. of Math. (2), 62:1 (1955), 8-43.

[2] Дж. Касселс, А. Фрёлих (ред.), Алгебрачческал теория чисел, Мир, М., 1969, 483 с.; пер. с англ.: J.W.S. Cassels, A. Fröhlich (eds.), Algebraic number theory, Proceedings of the instructional conference (Univ. of Sussex, Brighton, September 1-17, 1965), 2nd ed., Academic Press, Inc. [Harcourt Brace Jovanovich, Publishers], London, 2010, xviii+366 pp.

[3] V. Chernousov, V. Guletskiu, "2-torsion of the Brauer group of an elliptic curve: generators and relations", Proceedings of the conference on quadratic forms and related topics (Baton Rouge, LA, 2001), Doc. Math., 2001, Extra vol., 85-120 (electronic).

[4] V. I. Chernousov, A. S. Rapinchuk, I. A. Rapinchuk, "On the genus of a division algebra", C. R. Math. Acad. Sci. Paris, 350:17-18 (2012), 807-812.

[5] V. I. Chernousov, A.S. Rapinchuk, I. A. Rapinchuk, "The genus of a division algebra and the unramified Brauer group", Bull. Math. Sci., 3:2 (2013), 211-240.

[6] V. I. Chernousov, A. S. Rapinchuk, I. A. Rapinchuk, "Estimating the size of the genus of a division algebra", in preparation.

[7] V. I. Chernousov, A. S. Rapinchuk, I. A. Rapinchuk, "On algebraic groups having the same maximal tori", in preparation.

[8] J.-L. Colliot-Thélène, "Birational invariants, purity, and the Gersten conjecture", $K$-theory and algebraic geometry: connections with quadratic forms and division algebras (Santa Barbara, CA, 1992), Proc. Sympos. Pure Math., 58, Part 1, Amer. Math. Soc., Providence, RI, 1995, 1-64.

[9] J.-L. Colliot-Thélène, "Groupe de Chow des zéro-cycles sur les variétés p-adiques (d'après S. Saito, K. Sato et al.)", Séminaire Bourbaki, v. 2009/2010, Exp. № 1012, Astérisque, 339, Soc. Math. France, Paris, 2011, vii+30 pp.

[10] J.-L. Colliot-Thélène, S. Saito, "Zéro-cycles sur les variétés $p$-adiques et groupe de Brauer", Internat. Math. Res. Notices, 1996, № 4, 151-160.

[11] B. Conrad, "Reductive group schemes", Autour des schémas en groupes, École d'été "Schémas en groupes" [Group Schemes, A celebration of SGA3], v. I (Luminy, 2011), Panoramas et Synthèses, 42-43, Soc. Math. France, Paris, 2014, http://smf4.emath.fr/en/Publications/PanoramasSyntheses/2014/42-43/html/smf_panosynth_42-43.php; 2014, 390 pp., math.stanford.edu/ ${ }^{\sim}$ conrad/papers/luminysga3.pdf.

[12] B. Conrad, "Non-split reductive groups over $\mathbb{Z} "$, Autour des schémas en groupes, École d'été "Schémas en groupes" [Group Schemes, A celebration of SGA3], v. II (Luminy, 2011), Panoramas et Synthèses, Soc. Math. France, Paris (to appear); 2014, 67 pp., math.stanford.edu/ ${ }^{\sim}$ conrad/papers/redgpZ.pdf. 
[13] P. Deligne, Cohomologie étale, Séminaire de géométrie algébrique du Bois-Marie SGA $4 \frac{1}{2}$. Avec la collaboration de J. F. Boutot, A. Grothendieck, L. Illusie, J. L. Verdier, Lecture Notes in Math., 569, Springer-Verlag, Berlin-New York, 1977, iv+312 pp.

[14] M. Demazure, A. Grothendieck (eds.), Schémas en groupes, v. III: Structure des schémas en groupes réductifs, Séminaire de géométrie algébrique du Bois Marie 1962/64 (SGA 3). Dirigé par M. Demazure, A. Grothendieck, Lecture Notes in Math., 153, Springer-Verlag, Berlin-Heidelberg-New York, 1970, viii+529 pp.

[15] B. Farb, R. K. Dennis, Noncommutative algebra, Grad. Texts in Math., 144, Springer-Verlag, New York, 1993, xiv+223 pp.

[16] J.-M. Fontaine, "Il n'y a pas de variété abélienne sur $\mathbb{Z} "$, Invent. Math., 81:3 (1985), $515-538$.

[17] O. Forster, Lectures on Riemann surfaces, Grad. Texts in Math., 81, Springer-Verlag, New York-Berlin, 1981, viii+254 pp.

[18] K. Fujiwara, "A proof of the absolute purity conjecture (after Gabber)", Algebraic geometry 2000 (Azumino (Hotaka)), Adv. Stud. Pure Math., 36, Math. Soc. Japan, Tokyo, 2002, 153-183.

[19] S. Garibaldi, A.S. Rapinchuk, "Weakly commensurable $S$-arithmetic subgroups in almost simple algebraic groups of types B and C", Algebra Number Theory, 7:5 (2013), $1147-1178$.

[20] S. Garibaldi, D. Saltman, "Quaternion algebras with the same subfields", Quadratic forms, linear algebraic groups, and cohomology, Dev. Math., 18, Springer, New York, 2010, 225-238.

[21] P. Gille, T. Szamuely, Central simple algebras and Galois cohomology, Cambridge Stud. Adv. Math., 101, Cambridge Univ. Press, Cambridge, 2006, xii+343 pp.

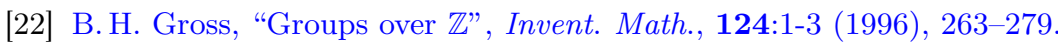

[23] O. T. Izhboldin, "Motivic equivalence of quadratic forms", Doc. Math., 3 (1998), 341-351 (electronic).

[24] M. Kac, "Can one hear the shape of a drum?", Amer. Math. Monthly, 73:4, Part 2 (1966), 1-23.

[25] N.A. Karpenko, "Criteria of motivic equivalence for quadratic forms and central simple agebras", Math. Ann., 317:3 (2000), 585-611.

[26] D. Krashen, E. Matzri, A. S. Rapinchuk, L. Rowen, D. Saltman, "Division algebras with common subfields", in preparation.

[27] D. Krashen, K. McKinnie, "Distinguishing division algebras by finite splitting fields", Manuscripta Math., 134:1-2 (2011), 171-182.

[28] С. Ленг, Основы диофантовой геометрии, Мир, М., 1986, 448 с.; пер. с англ.: S. Lang, Fundamentals of Diophantine geometry, Springer-Verlag, New York, 1983, xviii+370 pp.

[29] S. Lichtenbaum, "Duality theorems for curves over p-adic fields", Invent. Math., 7:2 (1969), 120-136.

[30] B. Linowitz, D. B. McReynolds, P. Pollack, L. Thompson, Counting and effective rigidity in algebra and geometry, 2014, 57 pp., arXiv: 1407.2294.

[31] C. Maclachlan, A. W. Reid, The arithmetic of hyperbolic 3-manifolds, Grad. Texts in Math., 219, Springer-Verlag, New York, 2003, xiv+463 pp.

[32] J.S. Meyer, "Division algebras with infinite genus", Bull. Lond. Math. Soc., 46:3 (2014), 463-468. 
[33] J.S. Milne, Class field theory, 2013, 281 pp.

www.jmilne.org/math/CourseNotes/cft.html.

[34] Р. Пирс, Ассочиативные алгебры, Мир, М., 1986, 543 с.; пер. с англ.: R. S. Pierce, Associative algebras, Grad. Texts in Math., 88, Stud. Hist. Modern Sci., 9, Springer-Verlag, New York-Berlin, 1982, xii+436 pp.

[35] В. П. Платонов, А. С. Рапинчук, Алгебраические группы и теория чисел, Наука, M., 1991, 656 с.; англ. пер.: V.P. Platonov, A.S. Rapinchuk, Algebraic groups and number theory, Pure Appl. Math., 139, Academic Press, Inc., Boston, MA, 1994, $\mathrm{xii}+614 \mathrm{pp}$.

[36] G. Prasad, A. S. Rapinchuk, "Weakly commensurable arithmetic groups and isospectral locally symmetric spaces", Publ. Math. Inst. Hautes Études Sci., 109 (2009), 113-184.

[37] G. Prasad, A. S. Rapinchuk, "On the fields generated by the lengths of closed geodesics in locally symmetric spaces", Geom. Dedicata, 172 (2014), 79-120.

[38] G. Prasad, A.S. Rapinchuk, "Generic elements in Zariski-dense subgroups and isospectral locally symmetric spaces", Thin groups and superstrong approximation, Math. Sci. Res. Inst. Publ., 61, Cambridge Univ. Press, Cambridge, 2014, 211-252.

[39] A.S. Rapinchuk, "Towards the eigenvalue rigidity of Zariski-dense subgroups", Proceedings of the International Congress of Mathematicians (Seoul, 2014), 247-269.

[40] A.S. Rapinchuk, I. A. Rapinchuk, "On division algebras having the same maximal subfields", Manuscripta Math., 132:3-4 (2010), 273-293.

[41] A. Reid, "Isospectrality and commensurability of arithmetic hyprebolic 2- and 3-manifolds", Duke Math. J., 65:2 (1992), 215-228.

[42] P. Roquette, The Brauer-Hasse-Noether theorem in historical perspective, Schriften der Mathematisch-naturwissenschaftlichen Klasse der Heidelberger Akademie der Wissenschaften, 15, Springer-Verlag, Berlin, 2005, vi+92 pp.

[43] D. J. Saltman, Lectures on division algebras, CBMS Regional Conf. Ser. in Math., 94, Amer. Math. Soc., Providence, RI; Conference Board of the Mathematical Sciences, Washington, DC, 1999, viii+120 pp.

[44] J.-P. Serre, Local fields, Grad. Texts in Math., 67, Springer-Verlag, New York-Berlin, 1979, viii+241 pp.

[45] Ж.П. Серр, Когомологии Галуа, Мир, М., 1968, 208 с.; пер. с фр.: J.-P. Serre, Cohomologie galoisienne, Cours au Collège de France, 1962-1963, 2-ème éd., Lecture Notes in Math., 5, Springer-Verlag, Berlin-Heidelberg-New York, 1964, vii+212 pp.; англ. пер.: J.-P. Serre, Galois cohomology, Springer Monogr. Math., Springer-Verlag, Berlin, 1997, x+210 pp.

[46] J. H. Silverman, The arithmetic of elliptic curves, 2nd ed., Grad. Texts in Math., 106, Springer, Dordrecht, 2009, xx+513 pp.

[47] S. V. Tikhonov, Division algebras of prime degree with infinite genus, 2014, 4 pp., arXiv: 1407.5041.

[48] E. B. Vinberg, Some examples of Fuchsian groups sitting in $S L_{2}(\mathbb{Q})$, preprint № 12011 of the SFB-701, Universität Bielefeld, Bielefeld, 2012, 4 pp., http://www.math.uni-bielefeld.de/sfb701/files/preprints/sfb12011.pdf.

[49] A. Vishik, Integral motives of quadrics, preprint MPI-1998-13, Max Planck Institute für Mathematik, Bonn, 1998, 82 pp., http://www.mpim-bonn.mpg.de/node/263. 
[50] A. Vishik, "Motives of quadrics with applications to the theory of quadratic forms", Geometric methods in the algebraic theory of quadratic forms, Lecture Notes in Math., 1835, Springer, Berlin, 2004, 25-101.

[51] A. R. Wadsworth, "Valuation theory on finite dimensional division algebras", Valuation theory and its applications, v. I (Saskatoon, SK, 1999), Fields Inst. Commun., 32, Amer. Math. Soc., Providence, RI, 2002, 385-449.

Владимир Иванович Черноусов (Vladimir I. Chernousov)

University of Alberta, Edmonton, Canada

E-mail: vladimir@ualberta.ca

\section{Андрей Степанович Рапинчук}

(Andrei S. Rapinchuk)

University of Virginia, Charlottesville, USA

E-mail: asr3x@virginia.edu

\section{Игорь Андреевич Рапинчук}

(Igor A. Rapinchuk)

Harvard University, Cambridge, USA

E-mail: rapinch@math.harvard.edu 